Research

Open Access

\title{
Diagnosis of ventilator-associated pneumonia: a systematic review of the literature
}

\author{
Alvaro Rea-Neto', Nazah Cherif M Youssef ${ }^{1}$, Fabio Tuche1, Frank Brunkhorst', V Marco Ranieri², \\ Konrad Reinhart ${ }^{1}$ and Yasser Sakr ${ }^{1}$
}

1Department of Anesthesiology and Intensive Care, Friedrich-Schiller-University Hospital, 07743 Jena, Germany
2Department of Anesthesiology and Intensive Care, S. Giovanni Battista Hospital, University of Turin, Turin, 10126, Italy

Corresponding author: Yasser Sakr, yasser.sakr@med.uni-jena.de

Received: 2 Feb 2008 Revisions requested: 26 Mar 2008 Revisions received: 1 Apr 2008 Accepted: 21 Apr 2008 Published: 21 Apr 2008

Critical Care 2008, 12:R56 (doi:10.1186/cc6877)

This article is online at: http://ccforum.com/content/12/2/R56

(c) 2008 Xiao et al.; licensee BioMed Central Ltd. This is an open access article distributed under the terms of the Creative Commons Attribution License (http://creativecommons.org/licenses/by/2.0), which permits unrestricted use, distribution, and reproduction in any medium, provided the original work is properly cited.

\begin{abstract}
Introduction Early, accurate diagnosis is fundamental in the management of patients with ventilator-associated pneumonia (VAP). The aim of this qualitative review was to compare various criteria of diagnosing VAP in the intensive care unit (ICU) with a special emphasis on the value of clinical diagnosis, microbiological culture techniques, and biomarkers of host response.
\end{abstract}

\begin{abstract}
Methods A MEDLINE search was performed using the keyword 'ventilator associated pneumonia' AND 'diagnosis'. Our search was limited to human studies published between January 1966 and June 2007. Only studies of at least 25 adult patients were included. Predefined variables were collected, including year of publication, study design (prospective/retrospective), number of patients included, and disease group.
\end{abstract}

Results Of 572 articles fulfilling the initial search criteria, 159 articles were chosen for detailed review of the full text. A total of 64 articles fulfilled the inclusion criteria and were included in our review. Clinical criteria, used in combination, may be helpful in diagnosing VAP, however, the considerable inter-observer variability and the moderate performance should be taken in account. Bacteriologic data do not increase the accuracy of diagnosis as compared to clinical diagnosis. Quantitative cultures obtained by different methods seem to be rather equivalent in diagnosing VAP. Blood cultures are relatively insensitive to diagnose pneumonia. The rapid availability of cytological data, including inflammatory cells and Gram stains, may be useful in initial therapeutic decisions in patients with suspected VAP. C-reactive protein, procalcitonin, and soluble triggering receptor expressed on myeloid cells are promising biomarkers in diagnosing VAP.

Conclusion An integrated approach should be followed in diagnosing and treating patients with VAP, including early antibiotic therapy and subsequent rectification according to clinical response and results of bacteriologic cultures.

\section{Introduction}

Ventilator-associated pneumonia (VAP) is common in the intensive care unit (ICU), affecting 8 to $20 \%$ of ICU patients and up to $27 \%$ of mechanically ventilated patients [1]. Several risk factors have been reported to be associated with VAP, including the duration of mechanical ventilation, and the presence of chronic pulmonary disease, sepsis, acute respiratory distress syndrome (ARDS), neurological disease, trauma, prior use of antibiotics, and red cell transfusions [2]. Mortality rates in patients with VAP range from 20 to $50 \%$ and may reach more than $70 \%$ when the infection is caused by multi-resistant and invasive pathogens [1-3]. The incidence of VAP-attributable mortality is difficult to quantify due to the possible confounding effect of associated conditions, but VAP is thought to increase the mortality of the underlying disease by about $30 \%$ [3]. VAP is also associated with considerable morbidity, including prolonged ICU length of stay, prolonged mechanical ventilation, and increased costs of hospitalization $[3,4]$.

ARDS $=$ Acute respiratory distress syndrome; $\mathrm{BAL}=$ Bronchoalveolar lavage; $\mathrm{CFU}=$ Colony forming units; $\mathrm{CPIS}=$ Clinical pulmonary infection score; $\mathrm{CRP}=\mathrm{C}$-reactive protein; $\mathrm{EF}=$ Elastin fiber; ICU = Intensive care unit; NNIS = National Nosocomial Infection Surveillance; $\mathrm{pBAL}=\mathrm{Protected}$ bronchoalvolar lavage; $\mathrm{PCT}=$ Procalcitonin; PSB = Protected specimen brush; SIRS = Systemic inflammatory response syndrome; sTREM = Soluble triggering receptor expressed on myeloid cells; TBA $=$ Tracheobronchial aspirate; VAP $=$ Ventilator-associated pneumonia. 
Delayed diagnosis and subsequent delay in initiating appropriate therapy may be associated with worse outcomes in patients with VAP $[1,5,6]$; on the other hand, an incorrect diagnosis may lead to unnecessary treatment and subsequent complications related to therapy $[1,7,8]$. Early, accurate diagnosis is, therefore, fundamental in the management of patients with VAP [9]. Several criteria have been proposed for diagnosing VAP in clinical settings, including clinical manifestations, imaging techniques, methods to obtain and interpret bronchoalveolar specimens, and biomarkers of host response. Due to the lack of an acceptable gold standard, the accuracy of these methods in diagnosing VAP is controversial.

The aim of this qualitative review was, therefore, to compare various criteria for diagnosing VAP in the ICU with a special emphasis on the value of clinical diagnosis, microbiological culture techniques, and biomarkers of host response.

\section{Materials and methods}

We performed a MEDLINE search using the keywords 'ventilator associated pneumonia' AND 'diagnosis'. Our search was limited to human studies published between January 1966 and June 2007. The abstracts of all articles were used to confirm our target population (patients with VAP) and the corresponding full-text articles were reviewed for the presence of data comparing a diagnostic test to a 'gold-standard'. Only studies of at least 25 adult patients were included. Two investigators (AR and NC) independently identified the eligible literature. Predefined variables were collected, including year of publication, study design (prospective/retrospective), number of patients included, and disease group. Any inconsistencies between the two investigators in interpretation of data were resolved by consensus. To avoid publication bias, abstracts and full articles were eligible. We also reviewed the bibliographies of available studies for other potentially eligible studies. Of 572 articles fulfilling the initial search criteria, 159 articles were chosen for detailed review of the full text. A total of 64 articles fulfilled the inclusion criteria and were included in our review.

\section{Results}

\section{Accuracy of the clinical diagnosis of VAP}

There is no single clinical manifestation that can be used alone to diagnose VAP. Chest radiology, although very sensitive, is typically nonspecific $[10,11]$. Wunderink et al. [12] showed that no roentgenographic sign correlates well with pneumonia in mechanically ventilated patients. Lobar or subsegmental atelectasia, ARDS, alveolar hemorrhage, and/or infarction may be mistaken for pneumonia [12]. Other clinical signs (fever, leukocytosis or pulmonary manifestations) have intermediate predictive values $[11,13]$. The clinical diagnosis of VAP has, therefore, traditionally been made by the association of a new or progressive consolidation on chest radiology plus at least two of the following variables: fever greater than $38^{\circ} \mathrm{C}$, leukocytosis or leukopenia, and purulent secretions. These criteria were proposed by Johanson et al. [14] (Table 1), and compared to immediate post-mortem lung biopsies by Fàbregas et al. [11]. The sensitivity was only $69 \%$ and specificity not better than $75 \%$ (accuracy of $72 \%$ ). An increase (or decrease) in the number of clinical criteria, can increase (or decrease) the specificity, but at the cost of sensitivity. Despite this relatively low accuracy, these criteria were recommended by the American Thoracic Society Consensus Conference on VAP [1].

Combinations of various criteria to establish a diagnosis in patients with VAP have been suggested and validated (Table 1). The National Nosocomial Infection Surveillance (NNIS) system was developed in the 1970s by the Centers for Disease Control as a tool to describe the epidemiology of hospital-acquired infections and to produce aggregated rates of infection suitable for inter-hospital comparison, but was never compared to pathological results. The NNIS system was compared to bronchoalveolar lavage (BAL) fluid cultures in 292 trauma patients and had a sensitivity of $84 \%$ and a specificity of $69 \%$ [15]. More recently, the Clinical Pulmonary Infection Score (CPIS) was proposed by Pugin et al. [16], based on six variables (fever, leukocytosis, tracheal aspirates, oxygenation, radiographic infiltrates, and semi-quantitative cultures of tracheal aspirates with Gram stain) [16]. The original description showed a sensitivity of $93 \%$ and specificity of $100 \%$, but this study included only 28 patients and the CPIS was compared to quantitative culture of BAL fluid using a 'bacterial index' defined as the sum of the logarithm of all bacterial species recovered, which is not considered an acceptable gold standard for the diagnosis of VAP. Compared to pathological diagnosis, CPIS had a moderate performance with a sensitivity between 72 and $77 \%$ and specificity between 42 and $85 \%$ $[11,17]$. Likewise, CPIS was not sufficiently accurate compared to a BAL fluid-established diagnosis with sensitivity between 30 and $89 \%$ and specificity between 17 and $80 \%$ [17-22] (Table 2). Luyt et al. [19] studied 201 mechanically ventilated patients in whom strict bronchoscopic criteria were applied to diagnose or exclude pneumonia. The CPIS assessed at baseline was calculated retrospectively and did not differ significantly for patients with or without VAP. The potential use of CPIS as the sole means to diagnosis VAP was also evaluated in 158 trauma patients [18]. The average CPIS was similar between patients with systemic inflammatory response syndrome (SIRS) (BAL $<10^{5}$ colony forming units (CFU)/ml) and those with VAP (BAL > $10^{5} \mathrm{CFUml}$ ) with a sensitivity of $61 \%$ and specificity of $43 \%$. In 28 patients with burn injuries, Pham et al. [23] found that CPIS had a sensitivity of $30 \%$ and specificity of $80 \%$ in diagnosing VAP compared to quantitative BAL fluid culture.

A major limitation of the literature validating CPIS for diagnosing VAP is that BAL culture is not a true gold standard [11,13,17,24-28]. In addition, the calculation of CPIS was modified by some authors and different cutoff points were used to diagnose VAP $[19,20]$. Importantly, the inter-observer 
Table 1

\section{Clinical criteria used in diagnosing ventilator-associated pneumonia}

Johanson criteria

- Presence of a new or progressive radiographic infiltrate

- Plus at least two of three clinical features:

- fever $>38^{\circ} \mathrm{C}$

- leukocyto sis or leukopeni

- purulent secretions

\begin{tabular}{|c|c|c|c|}
\hline & - Temperature & - Oxygenation ( $\mathrm{PaO} 2 / \mathrm{FiO} 2)$ & - Tracheal secretions (score) \\
\hline & - 0 point: $36.5-38.4 \mathrm{C}$ & - 0 point: $\mathrm{PaO} 2 / \mathrm{FiO} 2>240$ or ARDS & -0 point: $<14$ \\
\hline & - 1 point: $38.5-38.9$ & $\begin{array}{l}\text { - } 2 \text { points: } \mathrm{PaO} 2 / \mathrm{FiO} 2<240 \text { and no } \\
\text { evidence of ARDS }\end{array}$ & -1 point: $>14$ \\
\hline & -2 points: $<36$ or $>39$ & & -2 points: purulent sputum \\
\hline Clinical Pulmonary Infection & - Blood leukocytes (cells/ $\mu \mathrm{L}$ ) & & - Culture of tracheal aspirate \\
\hline & - 0 point: $4000-11000$ & & -0 point: minimal or no growth \\
\hline & - 1 point: $<4000$ or $>11000$ & & -1 point: moderate or more growth \\
\hline & - 2 points: $>500$ band forms & & -2 points: moderate or greater growth \\
\hline & & - Pulmonary radiography & \\
\hline & & -0 point: no infiltrate & \\
\hline & & - 1 point: diffuse or patchy infiltrates & \\
\hline & & - 2 points: localized infiltrate & \\
\hline & Total score of $>6$ points suggests ventilator-a & associated pneumonia & \\
\hline & $\mathrm{ARDS}=$ acute respiratory distress syndrome & & \\
\hline Centers for Disease Control and & - Radiology signs & - Clinical signs & \\
\hline & $\begin{array}{l}\text { Two or more serial chest radiographs with } \\
\text { at least } 1 \text { of the following: }\end{array}$ & At least 1 of the following: & \\
\hline & $\begin{array}{l}\text { - new or progressi ve and persistent } \\
\text { infiltrate }\end{array}$ & - fever (temperat ure $>38 \mathrm{C}$ ) & \\
\hline & - consolidation & $\begin{array}{l}\text { - leukopeni a (<4000 WBC) or leukocyto } \\
\text { sis }(>12000 \text { WBC) }\end{array}$ & \\
\hline & - cavitation & $\begin{array}{l}\text { - altered mental status, for adults } 70 \text { years } \\
\text { or older, with no other recognized cause }\end{array}$ & \\
\hline & - Microbiological criteria & & \\
\hline & At least one of the following: & Plus at least 2 of the following:- & \\
\hline & $\begin{array}{l}\text { - positive growth in blood culture not related } \\
\text { to another source of infection }\end{array}$ & $\begin{array}{l}\text { - new onset of purulent sputum, or change } \\
\text { in character of sputum }\end{array}$ & \\
\hline & - positive growth in culture or pleural field & $\begin{array}{l}\text { - increased respiratory secretions, or } \\
\text { increased suctioning requirements }\end{array}$ & \\
\hline & $\begin{array}{l}\text { - positive quantitati ve culture from } \\
\text { bronchoal veolar lavage }\left(>10^{4}\right) \text { or } \\
\text { protected specimen brushing }\left(>10^{3}\right)\end{array}$ & $\begin{array}{l}\text { - new-onset or worsening cough, or } \\
\text { dyspnea, or tachypnea }\end{array}$ & \\
\hline & $\begin{array}{l}\text { - five percent or more of cells with intracellul } \\
\text { ar bacteria on direct microsco pic examinati } \\
\text { on of Gram-stained bronchoal veolar lavage } \\
\text { fluid }\end{array}$ & - rales or bronchial sounds & \\
\hline & - histopathological evidence of pneumonia & - worsening gas exchange & \\
\hline & & - increased oxygen requirements & \\
\hline
\end{tabular}


Table 2

Studies comparing clinical criteria to other diagnostic (Dx) tests

\begin{tabular}{|c|c|c|c|c|}
\hline First author & Sample & Dx Tests & Gold standard & Results \\
\hline Fabregas, $1999,{ }^{\star \star}[11]$ & Medical ICU, 25 pts & $\begin{array}{l}\text { Johanson criteria, CPIS, } \\
\text { TBA }\left(10^{5}\right), \text { PSB }\left(10^{3}\right) \\
\operatorname{pBAL}\left(10^{4}\right), \operatorname{BAL}\left(10^{4}\right)\end{array}$ & Pathology + Culture & $\begin{array}{l}\text { - Johanson criteria ( } 2 \text { items): sens }=69 \%, \text { spec }=75 \% \text {. } \\
\text { - Any Johanson criteria: Chest Rx: sens }=92 \%, \text { spec }= \\
33 \% \text {; leukocytosis: sens }=77 \% \text {, spec }=58 \% \text {; fever: } \\
\text { sens }=46 \%, \text { spec }=42 \% ; \text { purulent secretions: sens }= \\
69 \%, \text { spec }=42 \% \\
\text { - CPIS: sens }=77 \%, \text { spec }=42 \% \text {. } \\
\text { - TBA sens }=69 \% \text {, spec }=92 \% \text {. } \\
\text { - pBAL sens }=39 \%, \text { spec }=100 \% \text {. } \\
\text { - BAL sens }=77 \%, \text { spec }=58 \% \text {. } \\
\text { - PSB sens }=62 \%, \text { spec }=75 \% \text {. } \\
\text { - QtC added little to clinical diagnostic accuracy }\end{array}$ \\
\hline Papazian, 1995, ** [17] & $\begin{array}{l}\text { Mixed ICU, } 38 \text { pts, } \\
\text { consecutive }\end{array}$ & $\begin{array}{l}\text { BBS }\left(10^{4}\right) \& \text { mini-BAL }\left(10^{3}\right) \& \\
\text { PSB }\left(10^{3}\right) \& \operatorname{BAL}\left(10^{4}\right) \& \\
\text { CPIS }\end{array}$ & Pathology + Culture & $\begin{array}{l}\text { - CPIS: sens }=72 \% ; \text { spec }=85 \% \\
\text { - BBS }\left(10^{4}\right): \text { sens }=83 \%, \text { spec }=80 \% \\
\text { - } \operatorname{mini}-B A L\left(10^{3}\right): \text { sens }=67 \%, \text { spec }=80 \% \\
\text { - BAL: sens }\left(10^{4}\right)=58 \%, \text { spec }=95 \% \\
\text { - PSB: sens }\left(10^{3}\right)=42 \%, \text { spec }=95 \% \\
\text { - BBS was more accurate than PSB }\end{array}$ \\
\hline Croce, 2006 * $\#$ \# [18] & Trauma ICU, 158 pts & CPIS $(>6)$ & $\mathrm{BAL}\left(10^{5}\right)$ & $\begin{array}{l}\text { - Frequency of VAP: } \mathrm{BAL} \geq 10^{5}=42 \%, \mathrm{SIRS}=58 \% \\
\text { - Average CPIS: VAP }=6.9, \mathrm{SIRS}=6.8 \\
\text { - CPIS }>6: \text { sens }=61 \%, \text { spec }=43 \%\end{array}$ \\
\hline Luyt, $20044^{*},{ }^{* *}[19]$ & Mixed ICU, 201 pts & CPIS $(>6)$ & $\begin{array}{l}\text { PSB }\left(10^{3}\right) \mathrm{BAL} \\
\left(10^{4}\right)\end{array}$ & $\begin{array}{l}\text { - CPIS: sens }=89 \%, \text { spec }=44 \%, k=0.33, P P V= \\
57 \%, N P V=84 \%\end{array}$ \\
\hline Schurink, 2004, ** [20] & Mixed ICU, 99 pts & CPIS & $\mathrm{BAL}\left(10^{4}\right)$ & $\begin{array}{l}- \text { Frequency of VAP }=69 \% \\
\text { - ROC curve for CIPS }>6,7 \text { and } 8=0.54,0.64,0.64 ; r \\
=0.115 \\
\cdot \text { CPIS }>5: \text { sens }=83 \%, \text { spec }=17 \% \\
\text { - CIPS }>6 \text { or } \leq 6: \mathrm{k} 0.16\end{array}$ \\
\hline Fartoukh, 2003, ** [21] & Mixed ICU, 68 pts & CE \& PIS $(>6)$ & $\begin{array}{l}\mathrm{BAL}\left(10^{4}\right) \text { or PTC } \\
\left(10^{3}\right)\end{array}$ & $\begin{array}{l}\text { - CE: sens }=50 \%, \text { spec }=59 \% \\
\text { - CPIS }>6: \text { sens }=60 \% \text {, spec }=59 \% \\
\text { - Adding positive Gram stain to CIPS improves } \\
\text { diagnostic accuracy }\end{array}$ \\
\hline Miller, 2006, ** [15] & Trauma ICU, 292 pts & NNIS & $\mathrm{BAL}\left(10^{5}\right)$ & $\begin{array}{l}\cdot \mathrm{k}=0.73 \\
\cdot \text { Sens }=84 \%, \mathrm{spec}=69 \%, \mathrm{PPV}=83 \%, \mathrm{NPV}=70 \%\end{array}$ \\
\hline Pham, 2007** ** [23] & Mixed ICU, 28 burn pts & CPIS & BAL & $\begin{array}{l}\text { - CPIS: sens }=30 \%, \text { spec }=80 \%, \text { PPV }=70 \%, \text { NPV }= \\
50 \%\end{array}$ \\
\hline Pugin, 1991, **, [16] & Surgical ICU, 28 pts, & CPIS \& mini-BAL $(B I \geq 5)$ & $\mathrm{BAL}(\mathrm{BI} \geq 5)$ & $\begin{array}{l}\text { - CPIS: sens }=93 \%, \text { spec }=100 \%, r=84 \%(\mathrm{CIPS} \text { and } \\
\text { mini-BAL), } r=76 \%(\mathrm{CPIS} \text { and BAL) } \\
\text { - Mini-BAL: sens }=73 \%, \text { spec }=96 \%\end{array}$ \\
\hline
\end{tabular}

$\mathrm{CPIS}=$ clinical pulmonary infection score; $\mathrm{TBA}=$ tracheobronchial aspirate; $\mathrm{PSB}=$ protected specimen brush; $\mathrm{PBAL}=$ protected $\mathrm{BAL}$ or 'mini-BAL'; $\mathrm{BAL}=$ bronchoalveolar lavage; sens = sensitivity; $s p e c=$ specificity; QtC = quantitative culture; $B B S=$ blind bronchial sampling; SIRS = systemic inflammatory response syndrome; $\mathrm{PPV}=$ positive predictive value; $\mathrm{NPV}=$ negative predictive value; $\mathrm{CE}=$ clinical estimate; $\mathrm{NNIS}=\mathrm{National}$ nosocomial infection surveillance system; $\mathrm{BI}=$ bacterial index; ICU $=$ intensive care unit; ${ }^{*}$ retrospective; ${ }^{* *}$ consecutive; ${ }^{\#}$ convenient.

agreement in calculating CPIS was found to be poor (kappa = $0.16)[20]$.

In summary: Clinical manifestations are usually used in combination with other features to diagnose VAP. Chest radiography may be sensitive but is typically nonspecific. NNIS criteria do not seem to be reliable for VAP diagnosis at the bedside. CPIS may be a helpful tool in diagnosing VAP, however, the considerable inter-observer variability and the moderate performance of the CPIS should be taken in account. Further studies are warranted to validate clinical criteria against pathological diagnosis.

\section{The role of bacteriological data in improving the accuracy of a clinical diagnosis of VAP}

Many studies have evaluated the value of bacteriological data in establishing the diagnosis of VAP compared to pathological, clinical, or other bacteriological diagnostic criteria (Tables $2,3,4,5,6)$. In a study by Torres et al. [26], quantitative cul- tures were obtained through BAL (bacterial count $=10^{4} \mathrm{CFU}$ ), protected BAL (pBAL) (104), protected specimen brush (PSB) $\left(10^{3}\right)$ and tracheobronchial aspirate (TBA) $\left(10^{5}\right)$ and were compared to five different histological and microbiological references [26]. Sensitivities for diagnosis of VAP ranged from $22 \%$ to $50 \%$ when only histologic reference tests were used, whereas specificity ranged from $45 \%$ to $100 \%$. When lung histology of guided or blind specimens and microbiology of lung tissue were combined [11,13,16,24-31], all quantitative diagnostic techniques achieved relatively higher, but still limited, diagnostic yields (sensitivity range 19\% to $87 \%$; specificity range $31 \%$ to $100 \%$ ). Fabregas et al. [11] also showed that addition of the results of quantitative cultures to clinical criteria (Johanson or CPIS) did not increase their accuracy in diagnosing VAP.

Quantitative cultures obtained by different methods, including $B A L, p B A L, P S B$ or TBA, seem to be rather equivalent in diagnosing VAP [22,31-41,41-58] (Table 4). Compared to a path- 
Table 3

Studies comparing quantitative cultures with pathology

\begin{tabular}{|c|c|c|c|c|}
\hline First author & Sample & Dx Tests & Gold standard & Results \\
\hline Balthazar, 2001, ** [13] & Mixed ICU, 37 pts & $\begin{array}{l}\text { BAL }\left(10^{4}\right) \& \text { Gram \& cells from } \\
\text { BAL }\end{array}$ & Pathology + Culture & $\begin{array}{l}\cdot \text { BAL: sens }=19 \%, \text { spec }=94 \% \text {; fever: sens }=50 \%, \text { spec } \\
=76 \% \text {; leucocytosis }(>10000): \text { sens }=60 \%, \text { spec }= \\
76 \% \text {; Gram stain: sens }=85 \% \text {, spec }=94 \% \text {; total cell } \\
(>400000): \text { sens }=90 \%, \text { spec }=94 \% .\end{array}$ \\
\hline Torres, $2000,{ }^{\star *}[26]$ & Medical ICU, 25 pts & $\begin{array}{l}\text { TBA }\left(10^{5}\right) \& \text { PSB }\left(10^{3}\right) \& \text { BAL } \\
\left(10^{4}\right) \& \text { pBAL }\left(10^{4}\right)\end{array}$ & Pathology + Culture & $\begin{array}{l}\text { - TBA: sens }=50 \%, \text { spec }=67 \% \\
\text { - PSB: sens }=67 \%, \text { spec }=75 \% \\
\text { - pBAL: sens }=63 \%, \text { spec }=83 \% \\
\text { - BAL: sens }=83 \%, \text { spec }=68 \%\end{array}$ \\
\hline Fabregas, $1999,{ }^{\star *}[11]$ & Mlxed ICU, 25 pts & $\begin{array}{l}\text { Johanson \& CPIS \& TBA (105), } \\
\text { PSB }\left(10^{3}\right), \operatorname{pBAL}\left(10^{4}\right) \text { and } \\
\text { BAL }\left(10^{4}\right)\end{array}$ & Pathology + Culture & $\begin{array}{l}\text { - Johanson criteria (2): sens }=69 \% \text {, spec }=75 \% \text {. } \\
\text { - Any Johanson criteria: Chest Rx: sens }=92 \%, \text { spec }= \\
33 \% \text {; leukocytosis: sens }=77 \% \text {, spec }=58 \% \text {; fever: sens } \\
=46 \%, \text { spec }=42 \% \text {; purulent secretions: sens }=69 \% \text {, } \\
\text { spec }=42 \% \text {. } \\
\text { - CPIS: sens }=77 \%, \text { spec }=42 \% \text {. } \\
\text { - TBA: sens }=69 \%, \text { spec }=92 \% \text {. } \\
\text { - pBAL: sens }=39 \% \text {, spec }=100 \% \text {. } \\
\text { - BAL: sens }=77 \%, \text { spec }=58 \% \text {. } \\
\text { - PSB: sens }=62 \%, \text { spec }=75 \% \text {. } \\
\text { - QtC increased little to clinical diagnosis accuracy. }\end{array}$ \\
\hline Papazian, 1997, \# [29] & Mixed ICU, 28 pts & Gram \& ICO & Pathology + Culture & $\begin{array}{l}\text { - BBS Gram stain: sens }=56 \% \text {, spec }=73 \% \text {. } \\
\text { - mini-BAL Gram Stain: sens }=44 \%, \text { spec }=87 \% \text {. } \\
\text { - BAL Gram stain: sens }=56 \%, \text { spec }=100 \% \\
\text { - BBS ICO }(>10 \%): \text { sens }=56 \% \text {, spec }=40 \% \text {. } \\
\text { - Mini-BAL ICO }(>5 \%): \text { sens }=67 \% \text {, spec }=53 \% \text {. } \\
\text { - BAL ICO }(>4 \%) \text { : sens }=56 \% \text {, spec }=40 \% \text {. }\end{array}$ \\
\hline Kirtland, 1997, ** [27] & Mixed ICU, 39 pts & $\begin{array}{l}\text { TA \&PSB \& pPSB \& BAL \& } \\
\text { BAL cells }\end{array}$ & Pathology + Culture & $\begin{array}{l}\text { - TA: sens }=87 \%, \text { spec }=31 \% \\
\text { - pPSB: sens }=30 \%, \text { spec }=81 \% \\
\text { - PSB: sens }=44 \%, \text { spec }=81 \% \\
\text { - BAL: sens }=65 \% \text {, spec }=63 \% \text {. } \\
\text { - }>50 \% \text { neutrophils in BAL: sens }=100 \%\end{array}$ \\
\hline Marquette, $1995,{ }^{\star *}[28]$ & Mixed ICU, 28 pts & $\begin{array}{l}\text { TA }\left(10^{5} \& 10^{6}\right) \& \operatorname{PSB}\left(10^{3}\right) \& \\
\operatorname{BAL}\left(10^{4}\right)\end{array}$ & Pathology & $\begin{array}{l}\text { - TA }\left(10^{5}\right): \text { sens }=63 \% \text {, spec }=75 \% \\
\text { - TA }\left(10^{6}\right): \text { sens }=50 \% \text {, spec }=85 \% \\
\text { - PSB }\left(10^{3}\right): \text { sens }=57 \% \text {, spec }=88 \% \\
\text { - BAL }\left(10^{4}\right): \text { sens }=47 \% \text {, spec }=100 \% \\
\text { - ICO }(\text { any } \%): \text { sens }=36 \% \text {, spec }=100 \%\end{array}$ \\
\hline Torres, $1996 *$ * * [25] & Mixed ICU, 25 pts & $\begin{array}{l}\operatorname{ICO}(\geq 5 \%), \operatorname{mini}-\mathrm{BAL}\left(10^{4}\right) \& \\
\operatorname{BAL}\left(10^{4}\right)\end{array}$ & Pathology & $\begin{array}{l}\text { - ICO }(\geq 5 \%) \text { compared to mini-BAL: PPV }=75 \%, \text { NPV = } \\
83 \% \text {. } \\
\text { - ICO }(\geq 5 \%) \text { compared to BAL: PPV }=57 \%, N P V=8 \\
3 \% \text {. } \\
\text { - Mini-BAL: sens }=22 \% \text {, spec }=100 \% \text {. } \\
\text { - BAL: sens }=45 \%, \text { spec }=55 \% .\end{array}$ \\
\hline Papazian, 1995, ** [17] & Mlxed ICU, 38 pts & $\begin{array}{l}\text { BBS }\left(10^{4}\right) \& \text { mini-BAL }\left(10^{3}\right) \& \\
\text { PSB }\left(10^{3}\right) \& \operatorname{BAL}\left(10^{4}\right) \& \\
\text { CPIS }\end{array}$ & Pathology + Culture & $\begin{array}{l}\text { - CPIS: sens }=72 \%, \text { spec }=85 \% \\
\text { - BBS }\left(10^{4}\right): \text { sens }=83 \%, \text { spec }=80 \% \\
\text { - } \operatorname{mini}-\mathrm{BAL}\left(10^{3}\right): \text { sens }=67 \%, \text { spec }=80 \% \text {. } \\
\text { - BAL }\left(10^{4}\right): \text { sens }=58 \%, \text { spec }=95 \% \\
\text { - } \operatorname{PSB}\left(10^{3}\right): \text { sens }=42 \%, \text { spec }=95 \%\end{array}$ \\
\hline Torres, 1994, ** [24] & Mixed ICU, 30 pts & $\begin{array}{l}\text { TBA }\left(10^{5}\right) \& \text { PSB }\left(10^{3}\right) \& \text { BAL } \\
\left(10^{4}\right) \& \text { Clinical data }\end{array}$ & Pathology & $\begin{array}{l}\text { - Clinical data: fever: sens }=55 \% \text {, spec }=58 \% \text {; purulent } \\
\text { secretions: sens }=83 \%, \text { spec }=33 \% \text {; Rx infiltrate: sens }= \\
78 \% \text {, spec }=42 \% \text {. } \\
\text { - Pulmonary biopsy culture }\left(\geq 10^{3}\right) \text { : sens }=40 \% \text {, spec }= \\
45 \% \text {. } \\
\text { - Quantitative cultures: TBA: sens }=44 \% \text {, spec }=48 \% \text {; } \\
\text { PSB: sens }=36 \%, \text { spec }=50 \% \text {; BAL: sens }=50 \% \text {, spec } \\
=45 \% \text {. }\end{array}$ \\
\hline Rouby, 1989, ** [30] & $\begin{array}{l}\text { Surgical ICU, } 59 \\
\text { pts }\end{array}$ & pBAL & Pathology + culture & $\begin{array}{l}\text { - pBAL: sens }=80 \%, \text { spec }=66 \% \text { to identify VAP; sens }= \\
73 \% \text { to identify the microorganism. }\end{array}$ \\
\hline Chastre, $1984,{ }^{* *}[10]$ & Mixed ICU, 26 pts & PSB $\left(10^{3}\right)$ & Lung culture & $\begin{array}{l}\text { - PSB correlated well to lung cultures, especially in the } \\
\text { subgroup of patients who received no antibiotics during } \\
\text { the week preceding their death. }\end{array}$ \\
\hline
\end{tabular}

$\mathrm{ICU}=$ intensive care unit; $\mathrm{BAL}=$ bronchoalveolar lavage; sens = sensitivity; spec = specificity; TBA = tracheobronchial aspirate; PSB = protected specimen brush; $\mathrm{pBAL}=$ protected $\mathrm{BAL}$ or 'mini-BAL'; CPIS = clinical pulmonary infection score; $\mathrm{QtC}=$ quantitative culture; ICO = intracellular organism; $\mathrm{BBS}=$ blind bronchial sampling; TA $=$ tracheal aspiration; $\mathrm{PPV}=$ positive predictive value; $\mathrm{NPV}=$ negative predictive value; ${ }^{*}=$ retrospective; ${ }^{* *}=$ consecutive; $\#$ convenient

ologically confirmed diagnosis of VAP, BAL (104) had a sensitivity between 19 and $83 \%$ and specificity between 45 and $100 \%$, PSB (103) a sensitivity between 36 and $83 \%$ and specificity between 50 and 95\%, pBAL (104) a sensitivity between 39 and $80 \%$ and specificity of 66 to $100 \%$, and TBA
(105) a sensitivity between 44 to $87 \%$ and specificity between 31 to $92 \%$ (Table 3) [10,11,13,17,24-30]. Torres et al. [26] demonstrated that prior antibiotic use considerably decreased the sensitivity of the cultures of BAL samples. Kirtland et al. [27] and Rouby et al. [30] reported that the microorganism 
Critical Care Vol 12 No 2 Rea-Neto et al.

Table 4

Studies comparing quantitative cultures using various techniques or cutoff points

\begin{tabular}{|c|c|c|c|c|}
\hline First author & Sample & Dx tests & Gold standard & Results \\
\hline Mondi, 2005, \# [31] & Trauma ICU, 39 pts & $\mathrm{TA}\left(10^{4}\right) \&\left(10^{5}\right)$ & BAL $\left(10^{5}\right)$ & $\begin{array}{l}\cdot \text { TA }\left(10^{4}\right): \text { sens }=95 \%, \text { spec }=58 \%, k=0.5339(p< \\
0.0001) . \\
\text { TA (105): sens }=90 \%, \text { spec }=68 \%, k=0.6384(p< \\
0.0001) .\end{array}$ \\
\hline $\begin{array}{l}\text { Brun-Buisson, 2005, ** } \\
\text { [32] }\end{array}$ & Mixed ICU, 68 pts & $\begin{array}{l}\text { TA (score } \geq 4+) \& \text { bPTC } \\
\left(10^{3}\right) \& \text { PTC }\left(10^{3}\right)\end{array}$ & $\begin{array}{l}\text { BAL }\left(10^{4} \text { or ICO }>\right. \\
2 \%)\end{array}$ & $\begin{array}{l}\text { - TA: sens }=77 \%, \text { spec }=81 \% \\
\text { - bPTC: } \text { sens }=77 \% \text {, spec }=97 \% \text {. } \\
\text { - PTC: sens }=77 \% \text {, spec }=94 \%\end{array}$ \\
\hline Davis, 2005, \# [42] & Trauma ICU, 155 pts & Gram (BAL) & $\mathrm{CDC}+\mathrm{BAL}\left(10^{5}\right)$ & $\begin{array}{l}\text { - Gram (BAL): sens }=88 \% \text { (any organism). } \\
\text { - Gram (BAL): sens }=73 \% \text {, spec }=49 \% ; P P V=78 \% \text {, } \\
\text { NPV }=42 \% \text {, accuracy }=65 \% \text { (Gram-negative). } \\
\text { - Gram (BAL): sens }=87 \% \text {, spec }=59 \% ; \text { PPV }=68 \% \text {, } \\
\text { NPV }=83 \% \text {, accuracy }=74 \% \text { (Gram-positive). }\end{array}$ \\
\hline Croce, $2004,{ }^{\star *}[43]$ & Trauma ICU, 526 pts & $\mathrm{BAL}\left(10^{4} \& 10^{5}\right)$ & BAL $\left(10^{5}\right)$ and ClEvol & $\begin{array}{l}\text { - BAL }\left(10^{5}\right): \text { sens }=95 \%, \text { spec }=10 \% \\
\text { - } \operatorname{BAL}\left(10^{4}\right): \text { sens }=99 \%, \text { spec }=70 \%\end{array}$ \\
\hline Miller, 2003, ** [44] & Trauma ICU, 168 pts & BAL $\left(10^{2}\right.$ to $\left.10^{4}\right)$ & $\mathrm{BAL}\left(10^{5}\right)$ & $\begin{array}{l}\text { - BAL }\left(10^{4} \text { and } 10^{3}\right) \text { : increased sensitivity }=14 \% \text {. } \\
\text { - BAL }\left(10^{2}\right) \text { : increased sensitivity }=16 \% \text {. }\end{array}$ \\
\hline Sirvent, 2003, ** [45] & Mixed ICU, 82 pts & $\mathrm{ICO}$ & Mini-BAL $\left(10^{3}\right)$ & $\begin{array}{l}\cdot I C O \geq 2 \%: \text { sens }=80 \%, \text { spec }=82 \% \\
\cdot I C O \geq 2 \% \text { better than } 1,5,7 \text { and } 10 \% .\end{array}$ \\
\hline $\mathrm{Wu}, 2002,{ }^{\star *}[35]$ & Medical ICU, 48 pts & $\mathrm{TA}\left(10^{5}\right)$ & $\begin{array}{l}\text { PSB }\left(10^{3}\right) \text { or BAL } \\
\left(10^{4}\right)\end{array}$ & $\begin{array}{l}\text { TA and PSB: sens }=91 \%, \text { spec }=72 \% ; \text { PPV }=75 \% \text {, } \\
\text { NPV }=90 \% \text {. } \\
\text { - TA and BAL: sens }=91 \%, \text { spec }=75 \% ; \text { PPV }=78 \% \text {, } \\
\text { NPV }=90 \% .\end{array}$ \\
\hline Duflo, 2001, ** [46] & Mixed ICU, 104 pts & Gram stain (mini-BAL) & Mini-BAL (103) & $\begin{array}{l}\text { - Gram stain: sens }=76 \%, \text { spec }=100 \%, \mathrm{k}=0.73 \text {, } \\
\text { concordance }=86 \%\end{array}$ \\
\hline Prekates, $1998,{ }^{*},{ }^{* *}[47]$ & & & BAL & $\begin{array}{l}\text { - Gram stain: sens }=77 \%, \text { spec }=87 \%, \text { PPV }=71 \% \text {, } \\
\text { NPV }=90 \%\end{array}$ \\
\hline Bello, 1996, *, ** [48] & ICU, 74 pts, consecutive & Mini-PSB & PSB and BAL & $\begin{array}{l}\text { - } \mathrm{BAL} \text { and PSB: concordance }=92 \% \text {. } \\
\text { - mini-PSB and BAL: concordance }=84 \% \text {. } \\
\text { - mini-PSB and PSB: concordance }=85 \% \text {. }\end{array}$ \\
\hline Pugin, 1991, ** [16] & Surgical ICU, 28 pts, & $\begin{array}{l}\text { CPIS \& mini-BAL }(B I \geq \\
\text { 5) }\end{array}$ & $\mathrm{BAL}(\mathrm{BI} \geq 5)$ & $\begin{array}{l}\text { - CPIS: sens }=93 \%, \text { spec }=100 \%, r=84 \%(\mathrm{CIPS} \text { and } \\
\text { mini-BAL), } r=76 \%(\mathrm{CPIS} \text { and BAL). } \\
\text { - Mini-BAL: sens }=73 \%, \text { spec }=96 \% \text {. }\end{array}$ \\
\hline Jourdain, 1995, ** [36] & Mixed ICU, 39 pts & TA $\left(10^{3}\right.$ to $\left.10^{7}\right)$ & $\begin{array}{l}\text { PSB }\left(10^{3}\right) \text { and ICO }(\geq \\
5 \%)\end{array}$ & $\begin{array}{l}\text { - TA }\left(10^{3}\right): \text { sens }=90 \% \text {, spec }=26 \% \text {, accuracy }=47 \% \text {. } \\
\text { - TA }\left(10^{4}\right): \text { sens }=84 \% \text {, spec }=40 \text {, accuracy }=54 \% \text {. } \\
\text { - TA }\left(10^{5}\right): \text { sens }=79 \% \text {, spec }=66 \% \text {, accuracy }=70 \% \text {. } \\
\text { - TA }\left(10^{6}\right): \text { sens }=68 \% \text {, spec }=84 \% \text {, accuracy }=79 \% \text {, } \\
\text { correlation }=40 \%(\text { TA and PSB). } \\
\text { - TA }\left(10^{7}\right): \text { sens }=21 \% \text {, spec }=92 \% \text {, accuracy }=68 \% \text {. }\end{array}$ \\
\hline Marik, 1995, ** [37] & Medical ICU, 53 pts & Mini-PSB $\left(10^{3}\right)$ & PSB (103) & - Mini-PSB and PSB: quantitative agreement $=85 \%$. \\
\hline Kollef, 1995, \# [38] & Medical ICU, 42 pts & $\begin{array}{l}\text { Mini-BAL }\left(10^{3}\right) \& \text { PSB } \\
\left(10^{3}\right)\end{array}$ & Johanson (ATS) & $\begin{array}{l}\text { - Mini-BAL: sens }=100 \%, \text { spec }=95 \% \text {. } \\
\text { - PSB: sens }=71 \% \text {, spec }=100 \% \text {. Good agreement } \\
\text { between mini-BAL and PSB cultures: } k=0,63 \text {, } \\
\text { concordance }=83 \% \text {. }\end{array}$ \\
\hline Rumbak, 1994, \# [39] & Mixed ICU, 38 pts & TA & PSB $\left(10^{3}\right)$ & $\begin{array}{l}\text { - TA: sens }=97 \%, \text { spec }=50 \%, \text { PPV }=91 \%, \text { NPV }= \\
80 \% .\end{array}$ \\
\hline Valles, $1994,{ }^{* \star}[49]$ & Mixed ICU, 42 pts & $I C O \& B A L$ & $\begin{array}{l}\text { Clinical criteria + PSB } \\
\left(10^{3}\right)\end{array}$ & 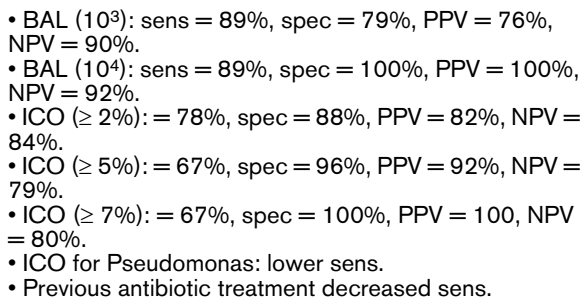 \\
\hline El-Ebiary, 1993, ** [40] & Medical ICU, 102 pts & TA, PSB and BAL & Clinical ad hoc & $\begin{array}{l}\text { - TA }\left(10^{5}\right): \text { sens }=70 \% \text {, spec }=72 \% \text {, accuracy }=71 \% \text {. } \\
\text { PSB }\left(10^{3}\right): \text { sens }=60 \% \text {, spec }=93 \text {, accuracy }=64 \% \text {. } \\
\text { - BAL }\left(10^{4}\right): \text { sens }=57 \% \text {, spec }=87 \% \text {, accuracy }= \\
67 \% .\end{array}$ \\
\hline Elatrous, 2004, ** [33] & Medical ICU, 100 pts, & TA $\left(10^{2}\right.$ to $\left.10^{6}\right)$ & PTC (106) & $\begin{array}{l}- \text { TA }\left(10^{2}\right): \text { sens }=96 \%, \text { spec }=66 \% \\
\text {-TA }\left(10^{3}\right): \text { sens }=94 \%, \text { spec }=66 \% \\
\text {-TA }\left(10^{4}\right): \text { sens }=92 \%, \text { spec }=85 \%, k=0,78 . \\
\text { - TA }\left(10^{5}\right): \text { sens }=84 \%, \text { spec }=90 \% \\
\text { - TA }\left(10^{6}\right): \text { sens }=44 \%, \text { spec }=94 \% .\end{array}$ \\
\hline
\end{tabular}


Table 4 (Continued)

Studies comparing quantitative cultures using various techniques or cutoff points

$\begin{array}{llll}\text { Mimoz, 2000, }{ }^{* *}[50] & \text { Mixed ICU, } 134 \text { pts, } & \begin{array}{l}\text { Gram stain (10 and } 50 \\ \text { fields) }\end{array} & \begin{array}{l}\text { PSB (103) or bPTC } \\ \left(10^{3}\right)\end{array} \\ & & & \\ \text { Flanagan, 2000, }{ }^{* *}[22] & \text { Mixed ICU, } 145 \text { pts, } & \begin{array}{l}\text { Mini-BAL (104) \& BAL } \\ (104) \& \text { PSB (103) \& } \\ \text { CPIS \& BI ( } \geq 5)\end{array} & \begin{array}{l}\text { Clinical (modified } \\ \text { CDC) }\end{array}\end{array}$

Allaouchiche, 1999, ** Mixed ICU, 118 pts, [51]

\begin{tabular}{|c|c|c|}
\hline Casetta, 1999, * ${ }^{*}[53]$ & $\begin{array}{l}\text { Mixed ICU (Cancer), } \\
42 \text { pts }\end{array}$ & PTC (103) \\
\hline $\begin{array}{l}\text { Souweine, 1998, ** } \\
\text { [54] }\end{array}$ & Mixed ICU, 52 pts & $\begin{array}{l}\text { Antibiotic use \& ICO } \\
(\geq 5 \%), \text { PSB }\left(10^{3}\right) \& \\
\text { BAL }\left(10^{5}\right)\end{array}$ \\
\hline
\end{tabular}

Allaouchiche, 1996, ${ }^{\star *}$ Mixed ICU, 132 pts
[52]

Barreiro, 1996, ${ }^{\star \star}[55] \quad$ Mixed ICU, 93 pts

Torres, 1989, ** [41] Mixed ICU, 34 pts

$\begin{array}{lll}\text { Timsit, 1993, }{ }^{* *}[56] & \text { Mixed ICU, 26 pts } & \text { PSB } 1 \\ \begin{array}{l}\text { Gerbeaux, 1998, }{ }^{* *} \\ {[57]}\end{array} & \text { Mixed ICU, 44 pts } & \text { BAL } 1 \\ \text { Butler, 2004, }{ }^{* \star}[58] & \text { Surgical ICU, 34 pts } & \text { Blind PSB } \\ \text { Wood, 2003, }{ }^{* *}[34] & \text { Trauma ICU, 90 pts } & \begin{array}{l}\text { TA \& BDPB \& BPB \& } \\ \text { BAL }\end{array}\end{array}$

ICO $(\geq 2 \%) \&$ Gram stain (BAL)

ICO

pBAL (104) \& Gram stain \& ICO $(\geq 2 \%)$

$B A L \& B I$

PTC \& BI

PSB (103)

Clin ad hoc

PSB $\left(10^{3}\right)+$ clinical evolution

PSB $\left(10^{3}\right)+$

Follow-up

PSB 2 (2 minutes interval)

BAL 2 (30 minutes interval)

Directed PSB
- Gram (10 fields) vs PSB: sens $=74 \%$, spec $=94 \%$. - Gram (10 fields) vs PTC: sens $=81 \%$, spec $=100 \%$ - PSB: correlation between morphology and culture: sens $=54 \%$, spec $=86 \%$.

- bPTC: correlation between morphology and culture: sens $=69 \%$ and $\mathrm{spec}=89 \%$.

- Mini-BAL: sens $=74 \%$, spec $=70 \%$, PPV $=17 \%$, $\mathrm{NPV}=96 \%$

- $\mathrm{BAL}:$ sens $=76 \%$, spec $=71 \%, \mathrm{PPV}=35 \%, \mathrm{NPV}=$ $93 \%$.

- PSB: sens $=68 \%$, spec $=86 \%$, PPV $=54 \%, N P V=$ $95 \%$.

- $\mathrm{CPIS}>7$ : sens $=85 \%$, spec $=91 \%$, PPV $=61 \%$,

NPV $=96 \%$
NPV

-BI: sens $=62 \%$, spec $=53 \%$.

- ICO: sens $=86 \%$, spec $=78 \%, P P V=68 \%$, $\mathrm{NPV}=91 \%, \mathrm{k}=0.616$, concordance $=81.5 \%$. - Gram stain: sens = 90\%, spec = 73\%, PPV = $64 \%, \mathrm{NPV}=91 \%, \mathrm{k}=0.58$, concordance = $79.4 \%$.

- Correlation between morphology and culture: complete $=51 \%$, partial $=39.2 \%$, no correlation $=9.8 \%$

- PTC: sens = 67\%, spec $=93 \% ;$ PPV $=71 \%$, $\mathrm{NPV}=91 \%$, agreement $=87 \%$.

- ICO: sens =71\% (no antibiotics), 50\% (current antibiotics), 67\% (recent antibiotics). - PSB: sens = 88\% (no antibiotics), 77\% (current antibiotics), 40\% (recent antibiotics). - BAL: sens $=71 \%$ (no antibiotics), 83\% (current antibiotics), $38 \%$ (recent antibiotics).

- $\operatorname{ICO}(\geq 2 \%):$ sens $=84 \%$, spec $=80 \%$, PPV $=$ $69 \%, \mathrm{NPV}=90 \%$, ROC $=0.888$.

- pBAL: sens $=87 \%$, spec $=91 \%$, PPV $=87 \%$ NPV $=91 \%$

- ICO: sens $=75 \%$, spec $=98 \%$, PPV = 96\%, $\mathrm{NPV}=86 \%$.

- BAL: spec $=71 \%, r=0,72$ (between $B A L$ and $\mathrm{BI})$ - PTC: spec $=86 \%, r=0.78$ (between PTC and $\mathrm{BI}$

- PSB 1: sens $=67 \%$, spec $=94 \%$.

-PSB 2: sens $=54 \%$, spec $=94 \%$.

- BAL 1-BAL2 repeatability $=75 \%$ with no bias, agreement $=47 \%$.

- Blind PSB and Directed PSB: concordance = $53 \%$.

- TA \& BAL: $k=0.535$

- BPB \& BDPB: $k=0.467$

- BPB \& BAL: $\mathrm{k}=0.547$.

$\mathrm{ICU}=$ intensive care unit; $\mathrm{TA}=$ tracheal aspiration; $\mathrm{BAL}=$ bronchoalveolar lavage; sens = sensitivity; spec $=$ specificity; $\mathrm{bPTC}=$ blinded protected telescoping catheter; PTC = protected (plugged) telescoping catheter; ICO = intracellular organism; CDC = center for disease control; $\mathrm{PPV}=$ positive predictive value; NPV = negative predictive value; CIEvol: clinical evolution; PSB = protected specimen brush; CPIS = clinical pulmonary infection score; $\mathrm{BI}=$ bacterial index; ATS = American Toracic Society; vs = versus; BDPB = bronchoscope-directed protected brushings; $\mathrm{BPB}=$ blind protected brushing via endotracheal tube; *retrospective; ${ }^{* *}$ consecutive; *convenient.

identified in quantitative culture from a transtracheal method frequently does not correlate well with the culture obtained from pathological samples. In addition, several studies [59-61] have challenged the reliability of quantitative cultures. Timsit et al. [56] reported moderate intra-individual variability in comparing two consecutive PSB procedures performed in the same lung. Likewise, Gerbeaux et al. [57] performed two BAL pro- cedures in the same lung area to distinguish between the presence and absence of bacterial pneumonia and showed a repeatability of only $75 \%$. Butler et al. [58] compared the results of blind PSB sampling from the contralateral lung and PSB from the site of the observed infiltrates; the concordance rate between the two samples was only $53 \%$. 
Table 5

Studies comparing quantitative cultures to clinical diagnosis

\begin{tabular}{|c|c|c|c|c|}
\hline First author & Sample & Dx tests & Gold standard & Results \\
\hline Camargo, 2004, ** [59] & Mixed ICU, 106 pts & $\begin{array}{l}\text { TA }\left(10^{5} \text { and } 10^{6}\right) \& \text { TA } \\
\text { (qualit) }\end{array}$ & Clinical (ad hoc) & $\begin{array}{l}-\mathrm{TA}\left(10^{6}\right): \text { sens }=26 \%, \text { spec }=78 \%, \mathrm{PPV}=20 \%, \mathrm{NPV} \\
=83 \% \\
\cdot \mathrm{TA}\left(10^{5}\right): \text { sens }=65 \%, \text { spec }=48 \%, \mathrm{PPV}=21 \%, \mathrm{NPV} \\
=87 \% \\
\text { - TA (qualit): sens }=81 \%, \text { spec }=23 \%, \mathrm{PPV}=18 \%, \\
\text { NPV }=86 \%\end{array}$ \\
\hline Mentec, $2004,{ }^{* *}[60]$ & Mixed ICU, 63 pts & $\begin{array}{l}\text { TA }\left(10^{5}\right), \text { bPTC }\left(10^{3}\right), \text { PTC } \\
\left(10^{3}\right), \text { BAL }\left(10^{4}\right)\end{array}$ & Clinical + Rx (ad hoc) & $\begin{array}{l}\text { - For quantitative cultures: TA: sens }=82 \%, \text { spec }= \\
67 \% \text {, ROC = 0,78; bPTC: sens }=62 \%, \text { spec }=94 \% \text {, } \\
\text { ROC =0,83; PTC: sens }=71 \%, \text { spec }=94 \%, \text { ROC }= \\
\text { 0,85; BAL: sens }=94 \%, \text { spec }=100 \%, \text { ROC }=0.98 \\
\text { - For Gram stain: TA: sens }=94 \%, \text { spec }=50 \% ; \text { bPTC: } \\
\text { sens }=56 \%, \text { spec }=94 \% ; \text { PTC } \text { sens }=65 \%, \text { spec }= \\
83 \% ; \text { BAL sens }=100 \%, \text { spec }=94 \%\end{array}$ \\
\hline Woske, 2001, ** [61] & $\begin{array}{l}\text { Surgical ICU, } 103 \text { pts, } \\
\text { consecutive }\end{array}$ & $\begin{array}{l}\text { BAL }\left(10^{4}\right) \& \text { PSB }\left(10^{3}\right) \& \\
\text { TA }\left(10^{5} \text { and } 10^{6}\right)\end{array}$ & CPIS $\geq 6$ & $\begin{array}{l}\text { - BAL: sens }=90 \% \\
\text { - PSB: sens }=83 \% \\
\text { - TA }\left(10^{5}\right): \text { sens }=90 \% \\
\text { - TA }\left(10^{6}\right): \text { sens }=50 \%\end{array}$ \\
\hline Meduri, 1992, ** [84] & Mixed ICU, 25 pts & pBAL (104) \& PSB & Clinical & - pBAL: $\mathrm{Spec}=100 \%, \mathrm{NPV}=100 \%$ \\
\hline Salata, 1987, ${ }^{\star \star}[85]$ & Mixed ICU, 51 pts & TA & Clinical & $\begin{array}{l}\text { - TA: higher Gram stain grading for neutrophils: } p<0.05 \\
\text { - TA: higher bacterial colony count: } p<0.05\end{array}$ \\
\hline Castro, 1991, ** [86] & Mixed ICU, 103 pts & PSB $\left(10^{3}\right)$ & Clinical & - PSB: sens $=84 \%$, spec $=67 \%$ \\
\hline
\end{tabular}

$\mathrm{ICU}=$ intensive care unit; $\mathrm{TA}=$ tracheal aspiration; qualit. = qualitative; $\mathrm{BAL}=$ bronchoalveolar lavage; sens $=$ sensitivity; spec $=$ specificity; $\mathrm{bPTC}=\mathrm{blinded}$ protected telescoping catheter; PTC = protected (plugged) telescoping catheter; $\mathrm{PPV}=$ positive predictive value; NPV = negative predictive value; $\mathrm{PSB}=$ protected specimen brush; CPIS = clinical pulmonary infection score; $\mathrm{pBAL}=$ protected bronchoalveolar lavage; ${ }^{\star \star}$ consecutive

The rate of positive blood cultures in VAP ranges from 8 to $20 \%[62,63]$. In 162 patients with suspected VAP, Luna et al. [64] showed that the sensitivity of blood cultures in 90 patients with VAP confirmed by BAL was only $26 \%$ and, in many cases, the bacteria isolated in the blood cultures probably had an extrapulmonary source. Blood cultures were also positive in 5 of 72 patients without VAP (6.9\%).

Since bacteriological cultures require some days for the results to be available at the bedside, several studies have investigated the value of cytological characteristics of the available bronchoalveolar specimens in diagnosing VAP. These characteristics include the number of inflammatory cells and Gram stains. The rapid availability of cytological data has been shown to be useful in initial therapeutic decisions in patients with suspected VAP $[42,46,47,50,51,65-67]$. The presence of more than $2 \%$ inflammatory cells had a sensitivity of $75 \%$ to $86 \%$ and a specificity of $78 \%$ to $98 \%$ in diagnosing the first episode of VAP (Table 6). Other studies $[46,49,50,68]$ investigated other cutoff values and reported contradictory results. Prior antibiotic use was also shown to influence the number of inflammatory cells in patients with VAP $[45,54]$. Infections with Pseudomonas aeruginosa were also shown to decrease the sensitivity of cytological data in diagnosing VAP compared with other bacteria [49]. The presence of bacteria in Gram stains of bronchoalveolar specimens had a sensitivity of $44 \%$ to $90 \%$ and specificity of $49 \%$ to $100 \%$ in identifying patients with VAP (Table 6). Davis et al. [42] showed that the accuracy of Gram stains was slightly better for Gram-positive than for Gram-negative microorganisms. Although the presence of bacteria on Gram stain appears to have a reasonable accuracy compared to quantitative culture available two to three days later, the agreement between the two methods ranges from 79.4 to $86 \%$ (Table 6).

In summary: Bacteriologic data do not increase the accuracy of diagnosis when compared to clinical diagnosis. Quantitative cultures obtained by different methods, including BAL, PBAL, PSB or TBA seem to be fairly equivalent in diagnosing VAP. Blood cultures are relatively insensitive to diagnose pneumonia. The rapid availability of cytological data, including inflammatory cells and Gram stains, may be useful in initial therapeutic decisions in patients with suspected VAP but may be influenced by prior antibiotic use and the infecting microorganism.

\section{Biomarkers and VAP diagnosis}

Many biological markers have been studied in an effort to improve the rapidity and performance of current diagnostic procedures in VAP. When the anatomical and mechanical defense mechanisms that prevent microorganisms from reaching alveoli are overwhelmed, a complex host response develops [1]. Microbial products activate alveolar macrophages, which release multiple endogenous mediators locally. Among these mediators, tumor necrosis factor alpha, interleukin-1 $\beta$, and other cytokines are increased in various types of pulmonary infections and thus have potential prognostic implications. However, there is no cutoff value for such mediators that could be used to diagnose pneumonia. Several biomarkers have been investigated for diagnosing VAP (Table 7). The presence of elastin fiber (EF), a marker of parenchymal lung destruction, in tracheal secretion has been proposed to differ- 
Table 6

Studies comparing cytological examination to quantitative cultures

\begin{tabular}{|c|c|c|c|c|}
\hline First author & Sample & Dx tests & Gold standard & Results \\
\hline Davis, 2005, \# [42] & Trauma ICU, 155 pts & Gram (BAL) & $\mathrm{CDC}+\mathrm{BAL}\left(10^{5}\right)$ & $\begin{array}{l}\text { - Gram (BAL): sens }=88 \% \text { (any organism). } \\
\text { - Gram (BAL): sens }=73 \% \text {, spec }=49 \%, \text { PPV }=78 \%, \text { NPV } \\
=42 \% \text {, accuracy }=65 \% \text { (Gram-negative). } \\
\text { - Gram (BAL): sens }=87 \% \text {, spec }=59 \%, \text { PPV }=68 \%, \text { NPV } \\
=83 \% \text {, accuracy }=74 \% \text { (Gram-positive). }\end{array}$ \\
\hline Sirvent, 2003, ${ }^{\star *}[45]$ & Mixed ICU, 82 pts & $\mathrm{ICO}$ & Mini-BAL $\left(10^{3}\right)$ & $\begin{array}{l}\cdot I C O \geq 2 \%: \text { sens }=80 \%, \text { spec }=82 \% \\
\cdot \text { ICO } \geq 2 \% \text { better than } 1,5,7 \text { and } 10 \%\end{array}$ \\
\hline Duflo, 2001, ** [46] & Mixed ICU, 104 pts & Gram stain (mini-BAL) & Mini-BAL $\left(10^{3}\right)$ & $\begin{array}{l}\text { - Gram stain: sens }=76 \%, \text { spec }=100 \%, \mathrm{k}=0.73 \text {, } \\
\text { concordance }=86 \%\end{array}$ \\
\hline Mimoz, 2000, ** [50] & Mixed ICU, 134 pts & $\begin{array}{l}\text { Gram stain (10 and } 50 \\
\text { fields) }\end{array}$ & $\begin{array}{l}\text { PSB }\left(10^{3}\right) \text { or bPTC } \\
\left(10^{3}\right)\end{array}$ & $\begin{array}{l}\text { - Gram stain (10 fields) vs PSB: sens }=74 \%, \text { spec }=94 \% \text {. } \\
\text { - Gram stain ( } 10 \text { fields) vs PTC: sens }=81 \%, \text { spec }=100 \% \text {. } \\
\text { - Gram ( } 50 \text { fields): slight increase in spec, decrease in sens } \\
\text { - Morphology and PSB culture: sens }=54 \%, \text { spec }=86 \% \text {. } \\
\text { - Morpholgy and bPTC culture: sens }=69 \%, \text { spec }=89 \% \text {. }\end{array}$ \\
\hline Allaouchiche, 1999, ** [51] & Mixed ICU, 118 pts & $\begin{array}{l}\operatorname{ICO}(\geq 2 \%) \& \text { Gram stain } \\
\text { (BAL) }\end{array}$ & PSB $\left(10^{3}\right)$ & $\begin{array}{l}\text { - ICO: sens }=86 \% \text {, spec }=78 \%, \mathrm{PPV}=68 \%, \mathrm{NPV}=91 \% \text {, } \\
\mathrm{k}=0.616, \text { concordance } 81.5 \% . \\
\cdot \text { Gram stain: sens }=90 \%, \text { spec }=73 \%, \mathrm{PPV}=64 \%, \mathrm{NPV}= \\
91 \% ; \mathrm{k}=0.58, \text { concordance } 79.4 \% . \\
\text { - Correlation between morphology and culture: complete: } \\
51 \% \text {, partial: } 39 . \% \text {, no correlation: } 9.8 \% .\end{array}$ \\
\hline Allaouchiche, 1996, ** [52] & Mixed ICU, 132 pts & $\mathrm{ICO}$ & $\begin{array}{l}\text { PSB }\left(10^{3}\right)+\text { clinical } \\
\text { evolution }\end{array}$ & $\begin{array}{l}\cdot \text { ICO }(\geq 2 \%): \text { sens }=84 \%, \text { spec }=80 \%, \text { PPV }=69 \%, \text { NPV } \\
=90 \%, \text { ROC }=0.888\end{array}$ \\
\hline Torres, $1996,{ }^{*},{ }^{* *}[25]$ & Mixed ICU, 25 pts & $\begin{array}{l}\text { ICO }(\geq 5 \%), \text { mini-BAL } \\
\left(10^{4}\right) \& B A L\left(10^{4}\right)\end{array}$ & Pathology & $\begin{array}{l}\text { - ICO }(\geq 5 \%) \text { compared to mini-BAL: PPV }=75 \%, N P V= \\
83 \% \text {. } \\
\text { - ICO ( } \geq 5 \%) \text { compared to BAL: PPV }=57 \%, N P V=83 \% \text {. } \\
\text { - Mini-BAL: sens }=22 \% \text {, spec }=100 \% \text {. } \\
\text { - BAL: sens }=45 \%, \text { spec }=55 \% \text {. }\end{array}$ \\
\hline Sole-Violan, 1994, ** [68] & Mixed ICU, З3 pts & $\begin{array}{l}\text { ICO (BAL) \& BAL }\left(10^{4}\right) \& \\
\text { PSB }\left(10^{3}\right)\end{array}$ & Clinical ad hoc & $\begin{array}{l}\text { - BAL: sens }=87 \%, \text { spec }=100 \% \\
\text { - PSB: sens }=75 \%, \text { spec }=100 \% \\
\text { - ICO }(>4 \%): \text { sens }=62 \%, \text { spec }=100 \%\end{array}$ \\
\hline Brasel, 2003, ** [66] & Surgical ICU, 35 pts & ICO 5\% \& ICO 7\% & $\mathrm{TA}\left(10^{4}\right) \& \mathrm{TA}\left(10^{5}\right)$ & $\begin{array}{l}\text { - ICO } 5 \% \text { and TA }\left(10^{4}\right): \text { sens }=61 \%, \text { spec }=89 \%, \text { PPV }= \\
90 \%, N P V=59 \%, \text { ROC }=0.84 . \\
\text { ICO } 5 \% \text { and TA }\left(10^{5}\right): \text { sens }=85 \%, \text { spec }=82 \%, \text { PPV }= \\
70 \%, N P V=91 \%, \text { ROC }=0.89 . \\
\text { ICO } 7 \% \text { and TA }\left(10^{4}\right): \text { sens }=39 \%, \text { spec }=97 \%, \text { PPV }= \\
96 \%, N P V=50 \%, \text { ROC }=0.86 . \\
\text { ICO } 7 \% \text { and TA }\left(10^{5}\right): \text { sens }=61 \%, \text { spec }=91 \%, \text { PPV }= \\
77 \%, N P V=82 \%, \text { ROC }=0.84 .\end{array}$ \\
\hline Timsit, 2001, ** [65] & Mixed ICU, 110 pts & $\begin{array}{l}\text { BAL-D ( } 1 \% \text { of infected } \\
\text { cells) }\end{array}$ & $\begin{array}{l}\mathrm{BAL}\left(10^{4}\right) \& \mathrm{PSB} \\
\left(10^{3}\right)\end{array}$ & $\begin{array}{l}\text { - BAL-D: sens }=93 \%, \text { spec }=91 \%, A U C=0.953, P P V= \\
90 \%, N P V=98 \%\end{array}$ \\
\hline Prekates, $1998,{ }^{* \star}[47]$ & $\begin{array}{l}\text { Surgical and Trauma } \\
\text { ICU, } 75 \text { pts, }\end{array}$ & Gram stain (BAL) & BAL & $\begin{array}{l}\text { - Gram stain: sens }=77 \%, \text { spec }=87 \%, \text { PPV }=71 \%, \text { NPV }= \\
90 \% \text {. }\end{array}$ \\
\hline
\end{tabular}

$\mathrm{ICU}=$ intensive care unit; $\mathrm{BAL}=$ bronchoalveolar lavage; $\mathrm{CDC}=$ center of disease control; sens = sensitivity; spec = specificity; PPV = positive predictive value; NPV $=$ negative predictive value; $\mathrm{ICO}=$ intracellular organism; PSB = protected specimen brush; $\mathrm{bPTC}=$ blinded protected telescoping catheter; $\mathrm{vs}=$ versus; $\mathrm{PTC}=$ protected (plugged) telescoping catheter; TA = tracheal aspiration; BAL-D = direct examination of BAL; *retrospective; ** consecutive; "* convenient.

entiate colonization from infection of the lung. However, the presence of EF in tracheal aspirates had low sensitivity (32\%) and only reasonable specificity (72\%) in diagnosing VAP [69]. In 22 patients with ARDS, Shepherd et al. [70] found that EF had a sensitivity of only $40 \%$ in diagnosing VAP. This may be due to the fact that EF correlates with lung destruction, more than infection per se.

Procalcitonin (PCT) and C-reactive protein (CRP) measurements have been shown to improve the clinical accuracy in identifying patients with SIRS caused by infection from SIRS of other causes. Serum PCT levels had a better performance than alveolar PCT concentrations, with a sensitivity of $41 \%$ and a specificity of $100 \%$ [71]. In patients after cardiac arrest and return of spontaneous circulation, PCT had a sensitivity of $100 \%$ and a specificity of $75 \%$ for the diagnosis of VAP [72].
Póvoa et al. showed that CRP (>9.6 mg/dl) had a good accuracy for VAP, with sensitivity of $87 \%$ and a specificity of $88 \%$ in a population of general ICU patients [73]. In addition, in 47 patients with microbiologically confirmed VAP, high CRP levels were associated with poor outcome [74].

The detection of soluble triggering receptor expressed on myeloid cells (sTREM)-1 in BAL fluid may be useful in establishing or excluding a diagnosis of bacterial or fungal pneumonia. Gibot et al. [75] studied 148 patients with suspected pneumonia and measured STREM-1 in their BAL fluid. These authors showed that the presence of STREM by itself was more accurate than any clinical findings or laboratory values in identifying the presence of bacterial or fungal pneumonia. Questions about the lack of a gold standard definition for VAP may have hampered classification of some of patients. In 28 
Table 7

Studies evaluating the value of biomarkers in diagnosing VAP

\begin{tabular}{|c|c|c|c|c|}
\hline First author & Sample & Dx Tests & Gold standard & Results \\
\hline Povoa, 2005, \# [73] & Mixed ICU, 112 pts & CRP & Johanson & $\begin{array}{l}\text { CRP }(>9.6 \mathrm{mg} / \mathrm{dl}): \text { sens }=87 \%, \text { spec }=88 \%, \text { AUC }= \\
0.92 .\end{array}$ \\
\hline Gibot, $2004,{ }^{\star *}[75]$ & Mixed ICU, 148 pts & sTREM-1 in mini-BAL & $\begin{array}{l}\text { Mini-BAL }\left(10^{3}\right) \text { Clinical (ad } \\
\text { hoc) }\end{array}$ & Sens $=98 \%$, spec $=90 \%$ \\
\hline Duflo, 2002, ** [71] & Mixed ICU, 96 pts & PCT serum \& alveolar & Mini-BAL (103) & $\begin{array}{l}\text { Serum PCT }(\geq 3.9 \mathrm{ng} / \mathrm{ml}): \text { sens }=41 \%, \mathrm{spec}=100 \% \text {, } \\
\text { AUC =0787. } \\
\text { Alveolar PCT: not useful. }\end{array}$ \\
\hline Oppert, 2002, ** [72] & Mixed ICU, 28 pts & PCT and PCR & Clinical (ad hoc) & Serum PCT $(>1 \mathrm{ng} / \mathrm{ml}):$ sens $=100 \%$, spec $=75 \%$ \\
\hline El-Ebiary, 1995, *, ** [69] & Mixed ICU, 78 pts & Elastin fibre & Clinical (ad hoc) & $E F:$ sens $=32 \%$, spec $=72 \%$ \\
\hline Determann, 2005, ** [76] & Mixed ICU, 28 pts & sTREM-1 & Clinical \& NBLF & Sens $=75 \%$, spec $=84 \%$ \\
\hline Pugin, 1992, ** [78] & Trauma ICU, 40 pts & BAL Endotoxin & Clinical \& BAL & $\begin{array}{l}\text { BAL endotoxin }>6 \mathrm{EU} / \mathrm{ml} \text { suggests pneumonia due to } \\
\text { Gram-negative bacteria. }\end{array}$ \\
\hline Flanagan, 2001, ** [77] & Mixed ICU, 64 pts & BAL Endotoxin & Clinical \& BAL & Sens $=81 \%$, spec $=87 \%$, PPV $=67 \%, N P V=95 \%$ \\
\hline
\end{tabular}

AUC: area under the curve; ICU = intensive care unit; $\mathrm{CRP}=\mathrm{C}$-reactive protein; sens = sensitivity; spec = specificity; sTREM-1 = soluble triggering receptor

expressed on myeloid cells; BAL = bronchoalveolar lavage; PCT = procalcitonin; EF = elastin fiber; NBLF = non-directed bronchial lavage fluids; EU = endotoxin units;

*retrospective; ${ }^{\star \star}$ consecutive; ${ }^{\#}$ convenient.

critically ill mechanically ventilated patients, Determann et al. [76] reported an increase in STREM-1 levels in non-directed BAL fluid obtained from patients who developed VAP $(n=9)$ in contrast to those who did not. A cutoff value for BAL fluid sTREM-1 levels of $200 \mathrm{pg} / \mathrm{ml}$ had a sensitivity of $75 \%$ and specificity of $84 \%$ in diagnosing pneumonia.

The value of endotoxin measurements in BAL fluid was also investigated, as approximately $70 \%$ of cases of VAP are caused by Gram-negative bacteria. Flanagan et al. [77] reported an increased concentration of endotoxin in bronchoscopic BAL and non-directed BAL fluid of patients with VAP. An endotoxin concentration of $6 \mathrm{EU} / \mathrm{ml}$ yielded the optimal operating characteristics (sensitivity of $81 \%$ and specificity of $87 \%$ ). In 40 samples of BAL fluid from patients with multiple trauma requiring prolonged mechanical ventilation, Pugin et al. [78] showed a relation between the concentration of endotoxin in lavage fluid and the quantity of Gram-negative bacteria. An endotoxin level greater than or equal to $6 \mathrm{EU} / \mathrm{ml}$ distinguished patients with Gram-negative bacterial pneumonia from colonized patients, and from those with pneumonia due to Gram-positive cocci.

The studies evaluating the value of the previously mentioned biomarkers are limited by the lack of a gold standard used to diagnosis VAP. EF and STREM-1 were compared to clinical diagnosis ad hoc $[75,76]$, PCT was compared to mini-BAL [71], and CRP to Johanson criteria [73].

In summary: CRP, PCT, and sTREM are promising biomarkers for diagnosing VAP, while EF and endotoxin concentrations are of limited value. Further studies are needed to fully determine the diagnostic accuracy of these and other biomarkers.

\section{Discussion}

Evaluating the performance of various diagnostic tests in patients with suspected VAP is challenging. The absence of a good gold standard for comparison is the main limiting factor in assessing these tests. Establishing a diagnosis of VAP, based on pathology or histology plus culture of the lung tissue, has a considerable degree of uncertainty, however, it is considered the best available gold standard [8]. Pathologic examination of lung tissue is not a perfect gold standard because patients who die are not representative of all patients with VAP. Moreover, many patients die after some days of antibiotic administration, which may alter results of bacteriologic analysis. In addition, pathology or tissue cultures may include nondiseased lung tissue leading to false negative results, and diagnostic criteria based on pathologic examination of lung tissue are not well defined [27,79]. Fabregas et al. [79] found that the histology and microbiology of post-mortem lung biopsies were poorly correlated, challenging the value of histological examination of lung tissue in diagnosing VAP.

The etiology of VAP probably involves microaspiration of secretions accumulating above the cuff of endotracheal tube [80]. These foci of microinfection may remain localized causing local bronchiolitis without clinically relevant pneumonia or proceed to develop into micro- or macro-bronchopneumonia. The multifocal, heterogeneous nature of VAP is one of the reasons why it is so difficult to establish a diagnosis of VAP. Biopsy specimens can miss the area of active disease. Alternatively, positive results may represent an area of clinically silent early bronchiolitis or resolving bronchial pneumonia. Likewise, cultures can miss the area of active disease (yielding false-negative results) or can detect clinically benign areas of bacterial colonization (yielding false positive results). In addition, the many antibiotics given to critically ill patients may decrease the diagnostic yield of bacterial cultures $[24,79]$. 
Associated cardiorespiratory comorbidities are another source of bias in diagnosing VAP. Autopsies of ventilated patients with suspected pneumonia frequently reveal a substantial burden of alternative or coexisting pulmonary diseases that can also cause fever, impaired gas exchange, increased secretions, and radiographic opacities. These other conditions include thromboembolic disease, hemorrhage, diffuse alveolar damage, fibrosis, atelectasia, carcinoma, lymphoma, and others $[11,12,24,27,28]$. The high prevalence of coexisting pulmonary diseases in ventilated patients further complicates attempts to clinically diagnose VAP.

The major limitation of the clinical approach to diagnosis is that it consistently leads to more antibiotic therapy than when therapy decisions are based on the findings of invasive lower respiratory tract samples. The clinical approach is overly sensitive, and patients can be treated for pneumonia when another noninfectious process is responsible for the clinical findings.

As none of the available diagnostic tests, performed alone, can provide an accurate diagnosis of VAP, a diagnostic strategy incorporating several criteria seems to be a good compromise. On the basis of clinical data, patients with clinical suspicion of VAP should be further evaluated by imaging procedures, bacteriological cultures, and biomarkers (Figure 1). The results of complementary diagnostic procedures should be used to refine the probability of diagnosing VAP and guide therapeutic decisions. Quantitative cultures should be performed on endotracheal aspirates or samples collected bronchoscopically, each technique having its own methodological limitations. Delays in the initiation of adequate antibiotic therapy increase mortality of VAP and thus therapy should not be postponed for the purpose of performing diagnostic studies in patients who are clinically unstable. The presence of organ dysfunction may necessitate the prompt initiation of antibiotic therapy. Garrard and A'Court [81] recommended regular, repeated surveillance with a simple, inexpensive, and well tolerated lavage technique in addition to daily clinical scoring to identify patients who may have VAP. A recent meta-analysis [82] of four randomized studies with a total of 628 patients showed that invasive strategies for the diagnosis of VAP did not alter mortality. In a recent multicenter trial, Heyland et al. [83] randomized 740 patients who were receiving mechanical ventilation and who had suspected VAP after 4 days in the ICU to undergo either BAL with quantitative culture of the BAL fluid or endotracheal aspiration with non-quantitative culture of the aspirate. Empirical antibiotic therapy was initiated in all patients until culture results were available, at which point a protocol of targeted therapy was used for discontinuing or reducing the dose or number of antibiotics, or for resuming antibiotic therapy to treat a pre-enrollment condition if the culture was negative. There was no significant difference in outcomes or the use of antibiotics. The most likely explanation for this lack of effect on outcome is that prompt adequate initial antimicrobial coverage is the crucial issue affecting survival. Inappropriate or inadequate treatment refers to the use of anti-

Figure 1

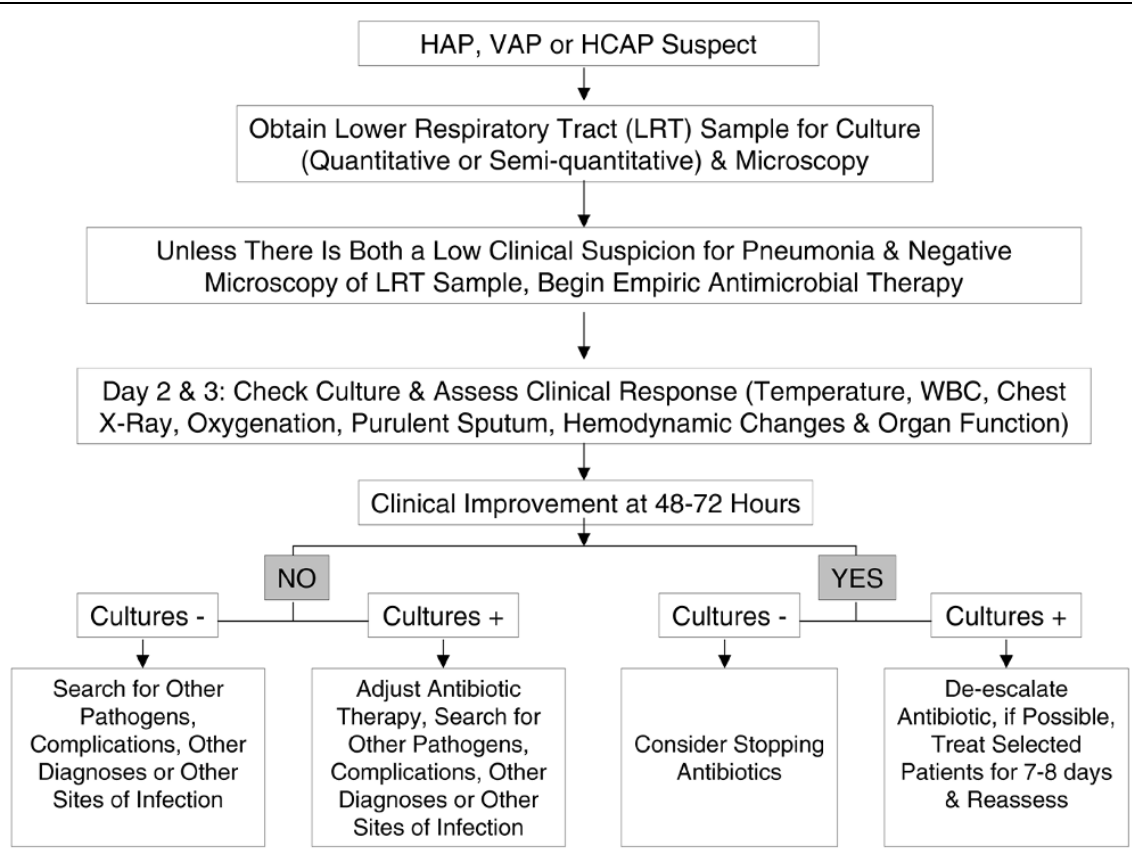

Summary of the management strategies for a patient with suspected hospital-acquired pneumonia (HAP), ventilator-associated pneumonia (VAP), or healthcare-associated pneumonia (HCAP). The decision about antibiotic discontinuation may differ depending on the type of sample collected (PSB, BAL, or endotracheal aspirate), and whether the results are reported in quantitative or semiquantitative terms. From [1] (with permission). 
biotics with either limited or no in vitro activity against the microorganism causing the infection. Since invasive sampling for suspected VAP does not directly affect the initial antibiotic prescription, it is not surprising that it does not alter mortality. Because of the nature of the technology, the culture results from bronchoscopy become available only after the crucial period when the clinician can intervene to maximal effect [82].

\section{Conclusion}

Clinical criteria, used in combination, may be helpful in diagnosing VAP; however, the considerable inter-observer variability and the moderate performance should be taken into account. Bacteriologic data do not increase the accuracy of diagnosis as compared to clinical diagnosis. Quantitative cultures obtained by different methods, including BAL, pBAL, PSB or TBA seem to be rather equivalent in diagnosing VAP. Blood cultures are relatively insensitive to diagnose pneumonia. The rapid availability of cytological data, including inflammatory cells and Gram stains, may be useful in initial therapeutic decisions in patients with suspected VAP. CRP, PCT, and sTREM are promising biomarkers in diagnosing VAP. An integrated approach should be followed in diagnosing and treating patients with VAP, including early antibiotic therapy and subsequent rectification according to clinical response and results of bacteriologic cultures.

\section{Key messages}

- Clinical criteria, used in combination, may be helpful in diagnosing VAP, however, the considerable interobserver variability and the moderate performance should be taken in account.

- Bacteriologic data do not increase the accuracy of diagnosis as compared to clinical diagnosis. Quantitative cultures obtained by different methods, including $B A L, p B A L, P S B$ or TBA seem to be rather equivalent in diagnosing VAP.

- The rapid availability of cytological data, including inflammatory cells and Gram stains, may be useful in initial therapeutic decisions in patients with suspected VAP.

- $\quad \mathrm{CRP}, \mathrm{PCT}$, and sTREM are promising biomarkers in diagnosing VAP.

- An integrated approach should be followed in diagnosing and treating patients with VAP, including early antibiotic therapy and subsequent rectification according to clinical response and results of bacteriologic cultures.

\section{Competing interests}

$\mathrm{KR}$ and FB have received fees from BRAHMS AG for speaking and for scientific advice. AR, NY, FT, and YS declare that they have no competing interests.

\section{Authors' contributions}

All authors participated in the design of the study. AR, NY, and FT contributed to data collection. AR, NY and YS drafted the manuscript. MR, KR and FB revised the article. All authors read and approved the final manuscript.

\section{Acknowledgements}

The study was supported by the German Federal Ministry of Education and Research (BMBF) Grant no. 01 KI 0106.

\section{References}

1. Guidelines for the management of adults with hospitalacquired, ventilator-associated, and healthcare-associated pneumonia. Am J Respir Crit Care Med 2005, 171:388-416.

2. Tejerina E, Frutos-Vivar F, Restrepo Ml, Anzueto A, Abroug F, Palizas F, González M, D'Empaire G, Apezteguía C, Esteban A, Internacional Mechanical Ventilation Study Group: Incidence, risk factors, and outcome of ventilator-associated pneumonia. J Crit Care 2006, 21:56-65.

3. Heyland DK, Cook DJ, Griffith L, Keenan SP, Brun-Bruisson C: The attributable morbidity and mortality of ventilator associated pneumonia in the critically ill patient. The Canadian Critical Trials Group. Am J Respir Crit Care Med 1999, 159:1249-1256.

4. Rello J, Ollendorf DA, Oster G, Vera-Llonch M, Bellm L, Redman $\mathrm{R}$, Kollef MH, VAP Outcomes Scientific Advisory Group: Epidemiology and outcomes of ventilator-associated pneumonia in a large US database. Chest 2002, 122:2115-2121.

5. Luna CM, Vujacich P, Niederman MS, Vay C, Gherardi C, Matera J, Jolly EC: Impact of BAL data on the therapy and outcome of ventilator-associated pneumonia. Chest 1997, 111:676-685.

6. Iregui M, Ward S, Sherman G, Fraser VJ, Kollef M: Clinical importance of delays in the initiation of appropriate antibiotic treatment for ventilator associated pneumonia. Chest 2002, 122:262-268

7. Deeks JJ: Systematic reviews in health care: systematic reviews of evaluations of diagnostic and screening tests. $B M J$ 2001, 323:157-162.

8. Klompas $\mathrm{M}$ : Does this patient have ventilator-associated pneumonia? JAMA 2007, 297:1583-1593.

9. Dellinger RP, Levy MM, Carlet JM, Bion J, Parker MM, Jaeschke R, Reinhart K, Angus DC, Brun-Buisson C, Beale R, Calandra T, Dhainaut JF, Gerlach H, Harvey M, Marini JJ, Marshall J, Ranieri M, Ramsay G, Sevransky J, Thompson BT, Townsend S, Vender JS, Zimmerman JL, Vincent JL: Surviving Sepsis Campaign guidelines for management of severe sepsis and septic shock. Crit Care Med 2004, 32:858-873.

10. Chastre J, Viau F, Brun P, Pierre J, Dauge MC, Bouchama A, Akesbi A, Gibert C: Prospective evaluation of the protected specimen brush for the diagnosis of pulmonary infections in ventilated patients. Am Rev Respir Dis 1984, 130:924-929.

11. Fàbregas N, Ewig S, Torres A, El-Ebiary M, Ramirez J, de La Bellacasa JP, Bauer T, Cabello H: Clinical diagnosis of ventilator associated pneumonia revisited: comparative validation using immediate post-mortem lung biopsies. Thorax 1999, 54:867-873.

12. Wunderink RG, Woldenberg LS, Zeiss J, Day CM, Ciemins J, Lacher DA: The radiologic diagnosis of autopsy-proven ventilator-associated pneumonia. Chest 1992, 101:458-463.

13. Balthazar AB, Von NA, De Capitani EM, Bottini PV, Terzi RG, Araujo S: Diagnostic investigation of ventilator-associated pneumonia using bronchoalveolar lavage: comparative study with a postmortem lung biopsy. Braz J Med Biol Res 2001, 34:993-1001.

14. Johanson WG Jr, Pierce AK, Sanford JP, Thomas GD: Nosocomial respiratory infections with gram-negative bacilli. The significance of colonization of the respiratory tract. Ann Intern Med 1972, 77:701-706.

15. Miller PR, Johnson JC III, Karchmer T, Hoth JJ, Meredith JW, Chang MC: National nosocomial infection surveillance system: from benchmark to bedside in trauma patients. J Trauma 2006, 60:98-103. 
16. Pugin J, Auckenthaler R, Mili N, Janssens JP, Lew PD, Suter PM: Diagnosis of ventilator-associated pneumonia by bacteriologic analysis of bronchoscopic and nonbronchoscopic 'blind' bronchoalveolar lavage fluid. Am Rev Respir Dis 1991, 143:1121-1129.

17. Papazian L, Thomas P, Garbe L, Guignon I, Thirion X, Charrel J, Bollet C, Fuentes P, Gouin F: Bronchoscopic or blind sampling techniques for the diagnosis of ventilator-associated pneumonia. Am J Respir Crit Care Med 1995, 152:1982-1991.

18. Croce MA, Swanson JM, Magnotti LJ, Claridge JA, Weinberg JA, Wood GC, Boucher BA, Fabian TC: The futility of the clinical pulmonary infection score in trauma patients. J Trauma 2006, 60:523-527.

19. Luyt CE, Chastre J, Fagon JY: Value of the clinical pulmonary infection score for the identification and management of ventilator-associated pneumonia. Intensive Care Med 2004, 30:844-852.

20. Schurink CA, Van Nieuwenhoven CA, Jacobs JA, RozenbergArska M, Joore HC, Buskens E, Hoepelman Al, Bonten MJ: Clinical pulmonary infection score for ventilator-associated pneumonia: accuracy and inter-observer variability. Intensive Care Med 2004, 30:217-224.

21. Fartoukh M, Maitre B, Honore S, Cerf C, Zahar JR, Brun-Buisson $\mathrm{C}$ : Diagnosing pneumonia during mechanical ventilation: the clinical pulmonary infection score revisited. Am J Respir Crit Care Med 2003, 168:173-179.

22. Flanagan PG, Findlay GP, Magee JT, lonescu A, Barnes RA, Smithies M: The diagnosis of ventilator-associated pneumonia using non-bronchoscopic, non-directed lung lavages. Intensive Care Med 2000, 26:20-30.

23. Pham TN, Neff MJ, Simmons JM, Gibran NS, Heimbach DM, Kelin MB: The clinical pulmonary infection score poorly predicts pneumonia in patients with burns. J Burn Care Res 2007, 28:76-79.

24. Torres A, el-Ebiary M, Padró L, Gonzalez J, de la Bellacasa JP, Ramirez J, Xaubet A, Ferrer M, Rodriguez-Roisin R: Validation of different techniques for the diagnosis of ventilator-associated pneumonia. Comparison with immediate postmortem pulmonary biopsy. Am J Respir Crit Care Med 1994, 149:324-331.

25. Torres A, El-Ebiary M, Fábregas N, González J, de la Bellacasa JP, Hernández C, Ramírez J, Rodriguez-Roisin R: Value of intracellular bacteria detection in the diagnosis of ventilator associated pneumonia. Thorax 1996, 51:378-384.

26. Torres A, Fabregas N, Ewig S, de la Bellacasa JP, Bauer TT, Ramirez J: Sampling methods for ventilator-associated pneumonia: validation using different histologic and microbiological references. Crit Care Med 2000, 28:2799-2804.

27. Kirtland SH, Corley DE, Winterbauer RH, Springmeyer SC, Casey KR, Hampson NB, Dreis DF: The diagnosis of ventilator-associated pneumonia: a comparison of histologic, microbiologic, and clinical criteria. Chest 1997, 112:445-457.

28. Marquette $\mathrm{CH}$, Copin MC, Wallet $F$, Neviere R, Saulnier F, Mathieu $D$, Durocher A, Ramon P, Tonnel AB: Diagnostic tests for pneumonia in ventilated patients: prospective evaluation of diagnostic accuracy using histology as a diagnostic gold standard. Am J Respir Crit Care Med 1995, 151:1878-1888.

29. Papazian L, Autillo-Touati A, Thomas P, Bregeon F, Garbe L, Saux $P$, Seite R, Gouin F: Diagnosis of ventilator-associated pneumonia: an evaluation of direct examination and presence of intracellular organisms. Anesthesiology 1997, 87:268-276.

30. Rouby JJ, Rossignon MD, Nicolas MH, Martin de Lassale E, Cristin $\mathrm{S}$, Grosset J, Viars P: A prospective study of protected bronchoalveolar lavage in the diagnosis of nosocomial pneumonia. Anesthesiology 1989, 71:679-685.

31. Mondi MM, Chang MC, Bowton DL, Kilgo PD, Meredith JW, Miller PR: Prospective comparison of bronchoalveolar lavage and quantitative deep tracheal aspirate in the diagnosis of ventilator associated pneumonia. J Trauma 2005, 59:891-895.

32. Brun-Buisson C, Fartoukh M, Lechapt E, Honoré S, Zahar JR, Cerf C, Maitre B: Contribution of blinded, protected quantitative specimens to the diagnostic and therapeutic management of ventilator-associated pneumonia. Chest 2005, 128:533-544.

33. Elatrous S, Boukef R, Ouanes Besbes L, Marghli S, Nooman S, Nouira S, Abroug F: Diagnosis of ventilator-associated pneumonia: agreement between quantitative cultures of endotracheal aspiration and plugged telescoping catheter. Intensive Care Med 2004, 30:853-858.
34. Wood AY, Davit AJ 2nd, Ciraulo DL, Arp NW, Richart CM, Maxwell RA, Barker DE: A prospective assessment of diagnostic efficacy of blind protective bronchial brushings compared to bronchoscope-assisted lavage, bronchoscope-directed brushings, and blind endotracheal aspirates in ventilatorassociated pneumonia. J Trauma 2003, 55:825-834.

35. Wu CL, Yang DI, Wang NY, Kuo HT, Chen PZ: Quantitative culture of endotracheal aspirates in the diagnosis of ventilatorassociated pneumonia in patients with treatment failure. Chest 2002, 122:662-668.

36. Jourdain B, Novara A, Joly-Guillou ML, Dombret MC, Calvat $S$, Trouillet JL, Gibert C, Chastre J: Role of quantitative cultures of endotracheal aspirates in the diagnosis of nosocomial pneumonia. Am J Respir Crit Care Med 1995, 152:241-246.

37. Marik PE, Brown WJ: A comparison of bronchoscopic vs blind protected specimen brush sampling in patients with suspected ventilator-associated pneumonia. Chest 1995, 108:203-207.

38. Kollef MH, Bock KR, Richards RD, Hearns ML: The safety and diagnostic accuracy of minibronchoalveolar lavage in patients with suspected ventilator-associated pneumonia. Ann Intern Med 1995, 122:743-748.

39. Rumbak MJ, Bass RL: Tracheal aspirate correlates with protected specimen brush in long-term ventilated patients who have clinical pneumonia. Chest 1994, 106:531-534.

40. El-Ebiary M, Torres A, González J, de la Bellacasa JP, García C, Jiménez de Anta MT, Ferrer M, Rodriguez-Roisin R: Quantitative cultures of endotracheal aspirates for the diagnosis of ventilator-associated pneumonia. Am Rev Respir Dis 1993, 148:1552-1557.

41. Torres A, Puig de la Bellacasa J, Xaubet A, Gonzalez J, RodriguezRoisin R, Jimenez de Anta MT: Diagnostic value of quantitative cultures of bronchoalveolar lavage and telescoping plugged catheters in mechanically ventilated patients with bacterial pneumonia. Am Rev Respir Dis 1989, 140:306-310.

42. Davis KA, Eckert MJ, Reed RL 2nd, Esposito TJ, Santaniello JM, Poulakidas S, Luchette FA: Ventilator-associated pneumonia in injured patients: do you trust your Gram's stain? J Trauma 2005, 58:462-466.

43. Croce MA, Fabian TC, Mueller EW, Maish GO 3rd, Cox JC, Bee TK, Boucher BA, Wood GC: The appropriate diagnostic threshold for ventilator-associated pneumonia using quantitative cultures. J Trauma 2004, 56:931-934.

44. Miller PR, Meredith JW, Chang MC: Optimal threshold for diagnosis of ventilator-associated pneumonia using bronchoalveolar lavage. J Trauma 2003, 55:263-267.

45. Sirvent JM, Vidaur L, Gonzalez S, Castro P, de Batlle J, Castro A, Bonet A: Microscopic examination of intracellular organisms in protected bronchoalveolar mini-lavage fluid for the diagnosis of ventilator-associated pneumonia. Chest 2003, 123:518-523.

46. Duflo F, Allaouchiche B, Debon R, Bordet F, Chassard D: An evaluation of the Gram stain in protected bronchoalveolar lavage fluid for the early diagnosis of ventilator-associated pneumonia. Anesth Analg 2001, 92:442-447.

47. Prekates A, Nanas S, Argyropoulou A, Margariti G, Kyprianou T, Papagalos E, Paniara O, Roussos C: The diagnostic value of gram stain of bronchoalveolar lavage samples in patients with suspected ventilator-associated pneumonia. Scand J Infect Dis 1998, 30:43-47.

48. Bello S, Tajada A, Chacón E, Villuendas MC, Senar A, Gascón M, Suarez FJ: 'Blind' protected specimen brushing versus bronchoscopic techniques in the aetiolological diagnosis of ventilator-associated pneumonia. Eur Respir J 1996, 9:1494-1499.

49. Vallés J, Rello J, Fernández R, Blanch L, Baigorri F, Mestre J, Matas $L$, Marín A, Artigas A: Role of bronchoalveolar lavage in mechanically ventilated patients with suspected pneumonia. Eur J Clin Microbiol Infect Dis 1994, 13:549-558.

50. Mimoz O, Karim A, Mazoit JX, Edouard A, Leprince S, Nordmann $P$ : Gram staining of protected pulmonary specimens in the early diagnosis of ventilator-associated pneumonia. $\mathrm{Br} J$ Anaesth 2000, 85:735-739.

51. Allaouchiche B, Jaumain $\mathrm{H}$, Chassard D, Bouletreau P: Gram stain of bronchoalveolar lavage fluid in the early diagnosis of ventilator-associated pneumonia. BrJ Anaesth 1999, 83:845-849.

52. Allaouchiche $B$, Jaumain $\mathrm{H}$, Dumontet $\mathrm{C}$, Motin J: Early diagnosis of ventilator-associated pneumonia. Is it possible to define a 
cutoff value of infected cells in BAL fluid? Chest 1996, 110:1558-1565.

53. Casetta M, Blot F, Antoun S, Leclercq B, Tancrède C, Doyon F, Nitenberg G: Diagnosis of nosocomial pneumonia in cancer patients undergoing mechanical ventilation: a prospective comparison of the plugged telescoping catheter with the protected specimen brush. Chest 1999, 115:1641-1645.

54. Souweine B, Veber B, Bedos JP, Gachot B, Dombret MC, Regnier $B$, Wolff M: Diagnostic accuracy of protected specimen brush and bronchoalveolar lavage in nosocomial pneumonia: impact of previous antimicrobial treatments. Crit Care Med 1998, 26:236-244.

55. Barreiro B, Dorca J, Manresa F, Catalá I, Esteban L, Verdaguer R, Gudiol F: Protected bronchoalveolar lavage in the diagnosis of ventilator-associated pneumonia. Eur Respir J 1996, 9:1500-1507.

56. Timsit JF, Misset B, Francoual S, Goldstein FW, Vaury P, Carlet J: Is protected specimen brush a reproducible method to diagnose ICU acquired pneumonia? Chest 1993, 104:104-108.

57. Gerbeaux $P$, Ledorav $V$, Boussuges A, Molenat $F$, Jean P, Sainty JM: Diagnosis of nosocomial pneumonia in mechanically ventilated patients: repeatability of the bronchoalveolar lavage. Am J Respir Crit Care Med 1998, 157:76-80.

58. Butler KL, Best IM, Oster RA, Katon-Benitez I, Lynn WW, Bumpers $\mathrm{HL}$ : Is bilateral protected specimen brush sampling necessary for the accurate diagnosis of ventilator-associated pneumonia? J Trauma 2004, 57:316-322.

59. Camargo LF, De Marco FV, Barbas CS, Hoelz C, Bueno MA, Rodrigues M Jr, Amado VM, Caserta R, Martino MD, Pasternak J, Knobel E: Ventilator associated pneumonia: comparison between quantitative and qualitative cultures of tracheal aspirates. Crit Care 2004, 8:R422-R430.

60. Mentec H, May-Michelangeli L, Rabbat A, Varon E, Le TF, Bleichner G: Blind and bronchoscopic sampling methods in suspected ventilator-associated pneumonia. A multicentre prospective study. Intensive Care Med 2004, 30:1319-1326.

61. Woske HJ, Roding T, Schulz I, Lode H: Ventilator-associated pneumonia in a surgical intensive care unit: epidemiology, etiology and comparison of three bronchoscopic methods for microbiological specimen sampling. Crit Care 2001, 5:167-173.

62. Blasi F, Cosentini R: Non invasive methods for the diagnosis of pneumonia. Eur Respir Mon 1997, 3:157-174.

63. Bryan CS, Reynolds KL: Bacteremic nosocomial pneumonia period analysis of 172 episodes from a single metropolitan area. Am Rev Respir Dis 1984, 129:668-671.

64. Luna CM, Videla A, Mattera J, Vay C, Famiglietti A, Vujacich P, Niederman MS: Blood cultures have limited value in predicting severity of illness and as a diagnostic tool in ventilator-associated pneumonia. Chest 1999, 116:1075-1084.

65. Timsit JF, Cheval C, Gachot B, Bruneel F, Wolff M, Carlet J: Usefulness of a strategy based on bronchoscopy with direct examination of bronchoalveolar lavage fluid in the initial antibiotic therapy of suspected ventilator associated pneumonia. Intensive Care Med 2001, 27:640-647.

66. Brasel KJ, Allen B, Edmiston C, Weigelt J: Correlation of intracellular organisms with quantitative endotracheal aspirate. J Trauma 2003, 54:141-146.

67. Prekates A, Nanas S, Argyropoulou A, Margariti G, Kryprianou T, Papagalos $E$ : The diagnostic value of gram stain of bronchoalveolar lavage samples in patients with suspected ventilator associated pneumonia. Scand J Infect Dis 1998, 30:43-47.

68. Sole-Violan J, Rodriguez De CF, Rey A, Martin-Gonzalez JC, Cabrera-Navarro P: Usefulness of microscopic examination of intracellular organisms in lavage fluid in ventilator-associated pneumonia. Chest 1994, 106:889-894.

69. el-Ebiary M, Torres A, González J, Martos A, Puig de la Bellacasa $J$, Ferrer M, Rodriguez-Roisin R: Use of elastin fibre detection in the diagnosis of ventilator associated pneumonia. Thorax 1995, 50:14-17.

70. Shepherd KE, Lynch KE, Wain JC, Brown EN, Wilson RS: Elastin fibers and the diagnosis of bacterial pneumonia in the adult respiratory distress syndrome. Crit Care Med 1995, 23:1829-1834.

71. Duflo F, Debon R, Monneret G, Bienvenu J, Chassard D, Allaouchiche B: Alveolar and serum procalcitonin: diagnostic and prog- nostic value in ventilator-associated pneumonia. Anesthesiology 2002, 96:74-79.

72. Oppert M, Reinicke A, Muller C, Barckow D, Frei U, Eckardt KU: Elevations in procalcitonin but not $\mathrm{C}$-reactive protein are associated with pneumonia after cardiopulmonary resuscitation. Resuscitation 2002, 53:167-170.

73. Póvoa P, Coelho L, Almeida E, Fernandes A, Mealha R, Moreira P Sabino $\mathrm{H}$ : C-reactive protein as a marker of infection in critically ill patients. Clin Microbiol Infect 2005, 11:101-108.

74. Póvoa P, Coelho L, Almeida E, Fernandes A, Mealha R, Moreira P, Sabino H: C-reactive protein as a marker of ventilator-associated pneumonia resolution: a pilot study. Eur Respir J 2005, 25:804-812.

75. Gibot S, Cravoisy A, Levy B, Bene MC, Faure G, Bollaert PE: Soluble triggering receptor expressed on myeloid cells and the diagnosis of pneumonia. N Engl J Med 2004, 350:451-458.

76. Determann RM, Millo JL, Gibot S, Korevaar JC, Vroom MB, Poll T van der, Garrard CS, Schultz MJ: Serial changes in soluble triggering receptor expressed on myeloid cells in the lung during development of ventilator-associated pneumonia. Intensive Care Med 2005, 31:1495-1500.

77. Flanagan PG, Jackson SK, Findlay G: Diagnosis of gram negative, ventilator associated pneumonia by assaying endotoxin in bronchial lavage fluid. J Clin Pathol 2001, 54:107-110.

78. Pugin J, Auckenthaler R, Delaspre O, van Gessel E, Suter PM: Rapid diagnosis of gram negative pneumonia by assay of endotoxin in bronchoalveolar lavage fluid. Thorax 1992, 47:547-549.

79. Fàbregas $\mathrm{N}$, Torres $\mathrm{A}$, El-Ebiary $\mathrm{M}$, Ramírez J, Hernández $\mathrm{C}$, González J, de la Bellacasa JP, de Anta J, Rodriguez-Roisin R: Histopathologic and microbiologic aspects of ventilator-associated pneumonia. Anesthesiology 1996, 84:760-771.

80. Rouby JJ, Martin De Lassale E, Poete P, Nicolas MH, Bodin L, Jarlier V, Le Charpentier Y, Grosset J, Viars P: Nosocomial bronchopneumonia in the critically ill. Histologic and bacteriologic aspects. Am Rev Respir Dis 1992, 146:1059-1066.

81. Garrard CS, A'Court CD: The diagnosis of pneumonia in the critically ill. Chest 1995, 108:17S-25S.

82. Shorr AF, Sherner JH, Jackson WL, Kollef MH: Invasive approaches to the diagnosis of ventilator-associated pneumonia: a meta-analysis. Crit Care Med 2005, 33:46-53.

83. Canadian Critical Care Trials Group: A randomized trial of diagnostic techniques for ventilator-associated pneumonia. $N$ Engl J Med 2006, 355:2619-2630.

84. Meduri GU, Wunderink RG, Leeper KV, Beals DH: Management of bacterial pneumonia in ventilated patients. Protected bronchoalveolar lavage as a diagnostic tool. Chest 1992, 101:500-508.

85. Salata RA, Lederman MM, Shlaes DM, Jacobs MR, Eckstein E, Tweardy D: Diagnosis of nosocomial pneumonia in intubated, intensive care unit patients. Am Rev Respir Dis 1987, 135:426-432.

86. Castro FR, Sole-Violan J, Capuz BL, Luna JC, Rodriguez BG, Alonso JLM: Reliability of the bronchoscopic protected catheter brush in the diagnosis of pneumonia in mechanically ventilated patients. Crit Care Med 1991, 19:171-175. 